\title{
A BRIDGING MECHANISM IN THE HOMOGENISATION OF BRITTLE COMPOSITES WITH SOFT INCLUSIONS
}

\author{
MARCO BARCHIESI, GIULIANO LAZZARONI, AND CATERINA IDA ZEPPIERI
}

\begin{abstract}
We provide a homogenisation result for the energy-functional associated with a purely brittle composite whose microstructure is characterised by soft periodic inclusions embedded in a stiffer matrix. We show that the two constituents as above can be suitably arranged on a microscopic scale $\varepsilon$ to obtain, in the limit as $\varepsilon$ tends to zero, a homogeneous macroscopic energy-functional explicitly depending on the opening of the crack.
\end{abstract}

Keywords: $\Gamma$-convergence, multiscale analysis, homogenisation, free-discontinuity problems, fracture mechanics.

2010 Mathematics Subject Class. 49J45, 74E30, 74G65, 74Q05, 74R99.

\section{INTRODUCTION}

Composites are materials that show heterogeneities on length scales that are much larger than the atomic scale but which are essentially homogeneous at macroscopic length scales. Engineered composites are widely used to improve structural performances; indeed with an appropriate choice of the microstructure they may efficiently combine the attributes of their constituents. For this reason, in recent decades there has been an ever increasing interest in the homogenisation theory of composites (and the consequent derivation of effective models) both in the mathematical and in the engineering communities.

The object of the present paper is the homogenisation of a brittle composite whose microstructure consists of soft (or compliant) inclusions periodically arranged in a stiffer matrix.

In the setting of linearised elasticity and antiplane shear, according to Griffith's theory, the microscopic energy corresponding to a displacement $u: \Omega \rightarrow \mathbb{R}$ (where $\Omega \subset \mathbb{R}^{2}$ is open, bounded, and represents the cross section of a cylindrical body) is given by

$$
F_{\varepsilon}(u)=\int_{\Omega \cap \varepsilon P}|\nabla u|^{2} \mathrm{~d} x+\delta_{\varepsilon} \int_{\Omega \backslash \varepsilon P}|\nabla u|^{2} \mathrm{~d} x+\mathcal{H}^{1}\left(S_{u}\right),
$$

where $\varepsilon>0$ is the length-scale of the microstructure, $\varepsilon P$ is the $\varepsilon$-scaled copy of a connected, $Q$ periodic, open set $P \subset \mathbb{R}^{2}$, with $Q=(-1 / 2,1 / 2)^{2}$, and $\delta_{\varepsilon} \rightarrow 0^{+}$is the elastic modulus of the soft material. Thus, $\Omega \cap \varepsilon P$ and $\Omega \backslash \varepsilon P$ represent, respectively, the stiff and the compliant constituent of the brittle composite $\Omega$ (see Figure 1); moreover these two constituents are characterised by the same toughness, which here is normalised to one. The energy $F_{\varepsilon}$ is given by the sum of three terms: two bulk energy contributions relative to the two constituents of $\Omega$, and a surface energy contribution accounting for the energy needed to open the crack. The latter is identified with the one-dimensional discontinuity set $S_{u}$ of a displacement $u$ assumed to belong to $S B V^{2}(\Omega)$, the space of special functions of bounded variation for which the microscopic energy $F_{\varepsilon}$ is finite for every fixed $\varepsilon$.

Date: August 9, 2016. 


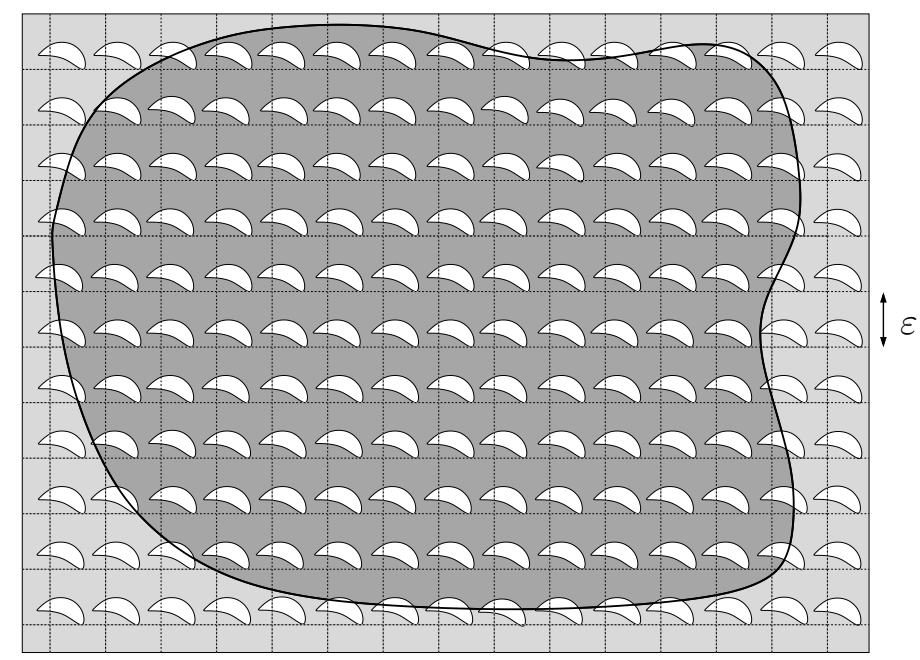

FiguRE 1. In dark grey the stiff matrix $\Omega \cap \varepsilon P$.

Using the tool of $\Gamma$-convergence $[9,17]$, in this paper we show that the limit behaviour of the functionals $F_{\varepsilon}$ is not, in general, a simple superposition of the corresponding limit behaviour of the bulk and surface energy terms. More precisely, we exhibit an elementary micro-geometry and an elastic modulus $\delta_{\varepsilon}$ for which optimal sequences of displacements may depend on the interplay between bulk and surface energy, thus favouring the presence of high gradients or discontinuities of $u$ inside the soft inclusions $\Omega \backslash \varepsilon P$. We show on a concrete example that even though the surface term in $F_{\varepsilon}$ does not depend on the opening of the crack, this dependence explicitly appears in the limit as $\varepsilon$ tends to zero.

Specifically, appealing to the localisation method of $\Gamma$-convergence and to the integral representation in $S B V$ [7], we prove that (up to subsequences) the functionals $F_{\varepsilon} \Gamma$-converge to a homogenised functional $F$ of the form

$$
F(u)=\int_{\Omega} f_{0}(\nabla u) \mathrm{d} x+\int_{S_{u}} g_{0}\left([u], \nu_{u}\right) \mathrm{d} \mathcal{H}^{1} \quad \text { for all } u \in S B V^{2}(\Omega),
$$

where $[u]$ denotes the opening of the crack and $\nu_{u}$ its orientation, while the energy densities $f_{0}$ and $g_{0}$ satisfy

$$
c_{1}|\xi|^{2} \leq f_{0}(\xi) \leq|\xi|^{2} \quad \text { and } \quad c_{2} \leq g_{0}(t, \nu) \leq 1
$$

for every $\xi \in \mathbb{R}^{2}, t \neq 0$, and $\nu \in \mathbb{S}^{1}$, and for some positive constants $c_{1}, c_{2}$. Moreover, $f_{0}$ depends only on $P$ (hence in particular not on $\delta_{\varepsilon}$ ) and it can be shown that $t \mapsto g_{0}(t, \nu)$ is nondecreasing and left-continuous for $t>0$, and satisfies the symmetry condition $g_{0}(-t,-\nu)=g_{0}(t, \nu)$.

The above $\Gamma$-convergence analysis strongly relies on some recent results for free-discontinuity problems in perforated domains $[22,14,5]$. In fact, the asymptotic analysis carried out in $[22,14,5]$ allows us to bound from below the functional $\Gamma$-liminf $F_{\varepsilon}$ with the Mumford-Shah functional, thus obtaining the equi-coerciveness of $F_{\varepsilon}$. (We now overlook the fact that the domain of $F$ is larger than $S B V^{2}(\Omega)$ and refer to Theorem 1 for a precise statement.)

The main result of this paper is Theorem 2 in which we specialise the functionals $F_{\varepsilon}$ choosing the two-dimensional micro-geometry given by

$$
P=\mathbb{R}^{2} \backslash \bigcup_{i \in \mathbb{Z}^{2}}\left(\frac{1}{4} \bar{Q}+i\right)
$$




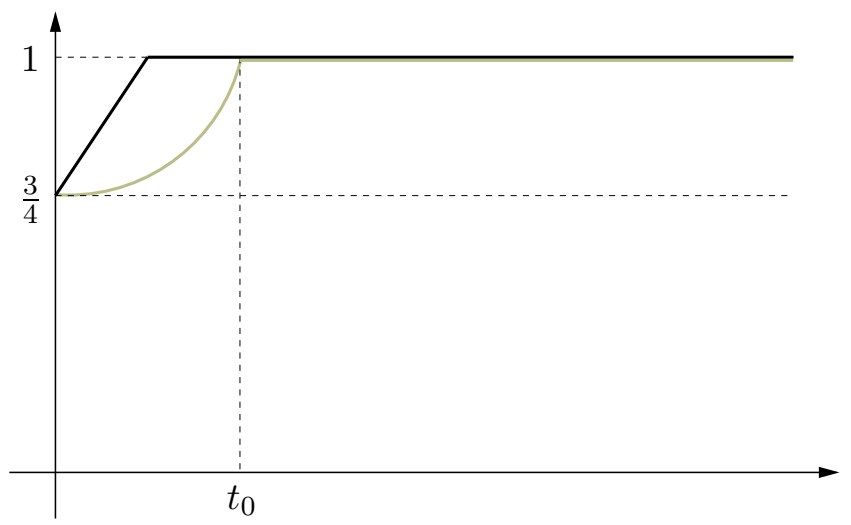

FiguRE 2. The surface energy density $g_{0}\left(\cdot, e_{i}\right)$ is bounded from above by $\min \{3 / 4+\sqrt{2} t, 1\}$ and from below by $\min \left\{3 / 4+c_{3} t^{2}, 1\right\}$. In particular, $g_{0}\left(0^{+}, e_{i}\right)=3 / 4$ while $g_{0}\left(t, e_{i}\right)=1$ for $t$ larger than a threshold $t_{0}$.

and setting $\delta_{\varepsilon}=\varepsilon$. We prove that for such a choice the following estimate holds true:

$$
\min \left\{\frac{3}{4}+c_{3} t^{2}, 1\right\} \leq g_{0}\left(t, e_{i}\right) \leq \min \left\{\frac{3}{4}+\sqrt{2} t, 1\right\} \quad \text { for } t>0 \text { and } i=1,2,
$$

where $g_{0}$ is as in (1.1). We mention here that the results which for modelling reasons have been described so far only in dimension two, can be suitably extended to the case of $n$-dimensional micro-geometries (and scalar displacement).

The estimate from below in (1.2) allows us to deduce that the surface energy density $g_{0}$ depends on $[u]$ in a nontrivial way. Moreover, the combination of the lower and upper bounds in (1.2) implies that $g_{0}\left(\cdot, e_{i}\right)$ is constant for every $t$ larger than a positive threshold $t_{0}$ (see Figure 2). For these reasons, our result can be also interpreted as a possible mesoscopic justification of cohesive zone energies; the latter being characterised by a surface energy density whose dependence on the crack-opening is nonconstant and nondecreasing for small openings, and bounded for large ones. Cohesive zone models have been introduced by Barenblatt in [6] and are widely employed in fracture mechanics since they provide a more accurate description of the process of crack growth if compared with those based on Griffith's criterion. Indeed, explicitly depending on the crack-opening $[u]$, they take into account that fracture is a gradual process due to the fact that atomic bonds stretch before breaking. In a discrete-to-continuum setting, cohesive surface energies have been derived by means of $\Gamma$-convergence in [10] starting from one-dimensional discrete systems and in [12] by mixing quadratic and defected springs. Moreover, cohesive-type models have been obtained in e.g. $[15,18]$ via Ambrosio-Tortorelli approximation and in [20] as limits of coupled elastoplastic-damage models. Further, in [23] it has been proved that cohesive energies converge to the Mumford-Shah functional if the size of the body tends to infinity.

The analysis carried out in the present paper is quite different: we show that a nontrivial dependence of the limit surface energy on the opening of the crack may arise from the interplay, on a mesoscopic scale, between two different brittle constituents, even though the micro-geometry is rather simple. We shall also mention that our limit model has a positive activation threshold $g_{0}\left(0^{+}, e_{i}\right)$. From a mechanical point of view it would be desirable to derive a cohesive model without activation threshold (or equivalently a limit surface density with $g_{0}\left(0^{+}, \nu\right)=0$ for every $\nu \in \mathbb{S}^{1}$ ) starting from an energy of brittle type; however, this seems to be a challenging question at the moment.

The presence of an activation threshold can be also found in a problem investigated in $[4,21]$, where a limit energy depending on the crack-opening is obtained homogenising a composite made 
of a brittle constituent reinforced by an unbreakable structure; i.e., by a structure with infinite toughness. It is worth to point out that in $[4,21]$ the limit surface energy density grows linearly in the crack-opening, thus showing an unphysical behaviour. Motivated by this observation, in the last section of this paper we briefly discuss a microscopic model similar to that analysed in $[4,21]$. We show that a cohesive model with bounded surface energy density can be obtained if the unbreakable structure is replaced by periodically distributed brittle fibres with toughness tending to infinity as the microscopic scale tends to zero.

Both in the presence of soft inclusions and of tough fibres, the general idea to prove the bound from below on the limit surface energy density is to approximate a straight crack, or in other words a limit displacement of type $u_{t}(x)=t \chi_{\left\{x: x_{2}>0\right\}}(x)$, with functions $u_{\varepsilon}$ suitably combining jumps and stretchings with high gradients in the different constituents of the composite (see Figures 5 and 10). Loosely speaking, at a microscopic level we observe (soft or tough) regions that stretch without breaking, thus acting as "bridges" between the two opposite sides of the macroscopic crack. This microscopic phenomenon is known in the mechanical literature as "bridging mechanism" and is experimentally observed e.g. in the fracture of fibre-reinforced plastics or ceramics and in the crazing of polymers [3, Chapter 6]: the crack propagation is preceded by the nucleation of micro-cracks and micro-voids, with some "bridging elements" that contribute in transferring stresses between the crack's faces, thus adding resistance to large crack-opening and to further growth.

\section{Setting of the Problem and statement of the main Result}

In this section we recall some definitions and introduce a few notation we employ in the paper. For the sake of generality we now work in dimension $n \geq 2$.

For the general theory of special functions of bounded variation we refer to [2] (see also [8]).

Let $U$ be an open bounded subset of $\mathbb{R}^{n}$. The space of special functions of bounded variation on $U$ is denoted by $S B V(U)$. For every $u \in S B V(U), \nabla u$ denotes the approximate gradient of $u, S_{u}$ the approximate discontinuity set of $u$, and $\nu_{u}$ the generalised normal to $S_{u}$, which is defined up to the sign. If $u^{+}$and $u^{-}$are the traces of $u$ on the sides of $S_{u}$ determined by $\nu_{u}$ and $-\nu_{u}$, respectively, the difference $u^{+}-u^{-}$is called the jump of $u$, and is denoted by $[u]$. Note that, with this convention, if we reverse the orientation of $\nu_{u}$, we change the sign of $[u]$. It turns out that $[u] \in L^{1}\left(S_{u} ; \mathcal{H}^{n-1}\right)$.

We consider the vector subspace of $S B V(U)$

$$
S B V^{2}(U):=\left\{u \in S B V(U): \nabla u \in L^{2}\left(U ; \mathbb{R}^{n}\right) \text { and } \mathcal{H}^{n-1}\left(S_{u}\right)<+\infty\right\} .
$$

We consider also the larger space of generalised special functions of bounded variation on $U$, $G S B V(U)$, which is made of all functions $u \in L^{1}(U)$ whose truncations $u^{m}:=(u \wedge m) \vee(-m)$ belong to $S B V(U)$ for every $m \in \mathbb{N}$. By analogy with the case of $S B V$ functions, we say that $u \in G S B V^{2}(U)$ if $u \in G S B V(U), \nabla u \in L^{2}\left(U ; \mathbb{R}^{n}\right)$ and $\mathcal{H}^{n-1}\left(S_{u}\right)<+\infty$.

For $r>0$ we denote by $Q_{r}$ the $n$-dimensional cube with side-length $r$, centred at the origin; i.e., $Q_{r}:=(-r / 2, r / 2)^{n}$; while we simply write $Q$ in place of $Q_{1}$.

The canonical basis in $\mathbb{R}^{n}$ is denoted by $\left\{e_{1}, \ldots, e_{n}\right\}$.

Unless otherwise stated, in what follows the $\Gamma$-convergence of functionals is always understood with respect to the strong $L^{1}$-topology, 
Let $\Omega$ be an open bounded subset of $\mathbb{R}^{n}$ and let $\delta_{\varepsilon}>0$ be such that $\delta_{\varepsilon} \rightarrow 0$ as $\varepsilon \rightarrow 0$. We introduce the functionals $F_{\varepsilon}: L^{1}(\Omega) \rightarrow[0,+\infty]$ defined as

$$
F_{\varepsilon}(u):= \begin{cases}\int_{\Omega \cap \varepsilon P}|\nabla u|^{2} \mathrm{~d} x+\delta_{\varepsilon} \int_{\Omega \backslash \varepsilon P}|\nabla u|^{2} \mathrm{~d} x+\mathcal{H}^{n-1}\left(S_{u}\right) & \text { if } u \in S B V^{2}(\Omega), \\ +\infty & \text { otherwise in } L^{1}(\Omega),\end{cases}
$$

where $P \subset \mathbb{R}^{n}$ is open, connected, and $Q$-periodic. We recall that in our model $\Omega$ represents the reference configuration of a periodic brittle composite made of two constituents having different elastic properties. More precisely, the elastic modulus of the constituent located in $\Omega \backslash \varepsilon P$ is represented by the vanishing sequence $\delta_{\varepsilon}$. For this reason, in what follows, $\Omega \backslash \varepsilon P$ is referred to as the soft inclusions. In order to keep our analysis as simple as possible, we assume that the two constituents have the same toughness, here normalised to one. However, straightforward computations show that analogous results also hold when the two constituents have different toughnesses independent of $\varepsilon$. For the case where the toughness of the soft inclusions scales as $\varepsilon^{\beta}$ with $\beta>0$, we refer to Remark 4 below.

We also consider the functionals $\hat{F}_{\varepsilon}: L^{1}(\Omega) \rightarrow[0,+\infty]$ given by

$$
\hat{F}_{\varepsilon}(u):= \begin{cases}\int_{\Omega \cap \varepsilon P}|\nabla u|^{2} \mathrm{~d} x+\mathcal{H}^{n-1}\left(S_{u} \cap \Omega \cap \varepsilon P\right) & \text { if }\left.u\right|_{\Omega \cap \varepsilon P} \in S B V^{2}(\Omega \cap \varepsilon P), \\ +\infty & \text { otherwise in } L^{1}(\Omega) .\end{cases}
$$

Notice that in this case $\Omega \backslash \varepsilon P$ represents a so-called perforation. The asymptotic behaviour of $\hat{F}_{\varepsilon}$ has been recently studied in [5, Theorem 4] (see also [14, 22]). Specifically, $\hat{F}_{\varepsilon} \Gamma$-converges with respect to the strong $L^{1}$-topology to

$$
\hat{F}(u):= \begin{cases}\int_{\Omega} \hat{f}(\nabla u) \mathrm{d} x+\int_{S_{u}} \hat{g}\left(\nu_{u}\right) \mathrm{d} \mathcal{H}^{n-1} & \text { if } u \in G S B V^{2}(\Omega), \\ +\infty & \text { otherwise in } L^{1}(\Omega),\end{cases}
$$

where $\hat{f}: \mathbb{R}^{n} \rightarrow[0,+\infty)$ and $\hat{g}: \mathbb{S}^{n-1} \rightarrow[0,+\infty)$ satisfy

$$
\begin{array}{cc}
c_{1}|\xi|^{2} \leq \hat{f}(\xi) \leq|\xi|^{2} & \text { for every } \xi \in \mathbb{R}^{n}, \\
c_{2} \leq \hat{g}(\nu) \leq 1 & \text { for every } \nu \in \mathbb{S}^{n-1},
\end{array}
$$

for some constants $c_{1}, c_{2}>0$ only depending on $n$ and $P$. Moreover, $\hat{f}$ is a quadratic form given by the following homogenisation formula:

$$
\hat{f}(\xi)=\inf \left\{\int_{Q \cap P}|\xi+\nabla w|^{2} \mathrm{~d} x: w \in H_{\mathrm{per}}^{1}(Q \cap P)\right\},
$$

where $H_{\mathrm{per}}^{1}(Q \cap P):=\left\{u \in H_{\mathrm{loc}}^{1}(P): u\right.$ is $Q$-periodic and $\left.\left.u\right|_{Q \cap P} \in H^{1}(Q \cap P)\right\}$. If $P$ is Lipschitz, the infimum in $(2.3)$ can be taken over $H_{\text {per }}^{1}(Q)$.

The convergence result as above is proved in [5] with respect to the strong $L^{2}$-topology; a technical but standard argument (see e.g. [21, Theorem 3.4]) allows us to deduce that the $\Gamma$-convergence of $\hat{F}_{\varepsilon}$ to $\hat{F}$ takes place also with respect to the strong $L^{1}$-topology.

The estimates in (2.2) and the fact that the domain of $\hat{F}$ is $G S B V^{2}(\Omega)$ are direct consequences of the compactness result provided in [5, Theorem 1], while (2.3) follows from [5, formula (40)].

It is also convenient to consider the Mumford-Shah functional

$$
M S(u):= \begin{cases}\int_{\Omega}|\nabla u|^{2} \mathrm{~d} x+\mathcal{H}^{n-1}\left(S_{u}\right) & \text { if } u \in G S B V^{2}(\Omega), \\ +\infty & \text { otherwise in } L^{1}(\Omega) .\end{cases}
$$


We notice that by virtue of the compactness result in $G S B V\left[2\right.$, Theorem 4.36] $M S$ is $L^{1}(\Omega)$ lower semicontinuous on $G S B V^{2}(\Omega)$.

We remark that

$$
\hat{F}_{\varepsilon} \leq F_{\varepsilon} \leq M S \text { on } S B V^{2}(\Omega),
$$

while, in view of the bounds from below in $(2.2)$, we get

$$
\min \left\{c_{1}, c_{2}\right\} M S \leq \hat{F} \quad \text { on } L^{1}(\Omega) .
$$

The bounds (2.4) and (2.5) will be crucial in the proof of the homogenisation result below.

Theorem 1. For every decreasing sequence of positive numbers converging to zero, there exists a subsequence $\left(\varepsilon_{k}\right)$ such that $\left(F_{\varepsilon_{k}}\right) \Gamma$-converges to a functional $F: L^{1}(\Omega) \rightarrow[0,+\infty]$ of the form

$$
F(u):= \begin{cases}\int_{\Omega} f_{0}(\nabla u) \mathrm{d} x+\int_{S_{u}} g_{0}\left([u], \nu_{u}\right) \mathrm{d} \mathcal{H}^{n-1} & \text { if } u \in G S B V^{2}(\Omega), \\ +\infty & \text { otherwise in } L^{1}(\Omega),\end{cases}
$$

where $f_{0}: \mathbb{R}^{n} \rightarrow[0,+\infty)$ coincides with the quadratic form $\hat{f}$ defined in $(2.3)$, and $g_{0}: \mathbb{R} \times$ $\mathbb{S}^{n-1} \rightarrow[0,+\infty)$ is a Borel function satisfying the following properties:

(i) for every $t \neq 0$ and $\nu \in \mathbb{S}^{n-1}, c_{2} \leq g_{0}(t, \nu) \leq 1$, where $c_{2}>0$ is as in (2.2);

(ii) for any $\nu \in \mathbb{S}^{n-1}, g_{0}(\cdot, \nu)$ is nondecreasing and left-continuous in $(0,+\infty)$ and satisfies the symmetry condition $g_{0}(-t,-\nu)=g_{0}(t, \nu)$.

The proof of Theorem 1 is contained in Section 3.

Remark 1. The assumption that the matrix $P$ is open and connected is needed to invoke the results in [5]. Specifically, the proof of Theorem 1 strongly relies on the fact that $\hat{F}=\Gamma-\lim \hat{F}_{\varepsilon}$ as well as on the bound (2.5). The latter, in its turn, together with (2.4), allows us to deduce that the domain of $F$ is $G S B V^{2}(\Omega)$.

Remark 2. The quadratic form $f_{0}$ does not depend on either the elastic modulus $\delta_{\varepsilon}$ or the surface term in $F_{\varepsilon}$.

The homogenisation result Theorem 1 asserts that the $\Gamma$-limit $F$ may depend on both $[u]$ and $\nu_{u}$, which in our modelling represent the opening and the orientation of the crack, respectively. In Theorem 2 below we show that for $\delta_{\varepsilon}=\varepsilon$ we can find an elementary two-dimensional microgeometry $\varepsilon P$ (see Figure 3 ) leading to a homogenised functional $F$ whose surface energy density $g_{0}$ actually depends on the crack-opening $[u]$. We notice that the choice $\delta_{\varepsilon}=\varepsilon$ provides the only meaningful scaling for the microgeometry under consideration, indeed other choices of $\delta_{\varepsilon}$ give no cohesive behaviour in the limit, see Remark 4 below.

For the sake of simplicity, we prove the dependence of $g_{0}$ on $[u]$ only when the normal to the crack $\nu$ is one of the coordinate vectors. Moreover, we state the result in the physically relevant case of dimension two; for higher dimension, see Remark 5 below.

The following theorem is the main result of this paper and is proved in Section 4.

Theorem 2. Let $n=2$; set $\delta_{\varepsilon}:=\varepsilon$ and

$$
P:=\mathbb{R}^{2} \backslash \bigcup_{(i, j) \in \mathbb{Z}^{2}} \bar{Q}_{\frac{1}{4}}+(i, j) .
$$

Then for every $t>0$ the energy density $g_{0}$ appearing in (2.6) satisfies the following growth conditions

$$
\min \left\{\frac{3}{4}+c_{3} t^{2}, 1\right\} \leq g_{0}\left(t, e_{i}\right) \leq \min \left\{\frac{3}{4}+\sqrt{2} t, 1\right\} \quad \text { for } i=1,2,
$$




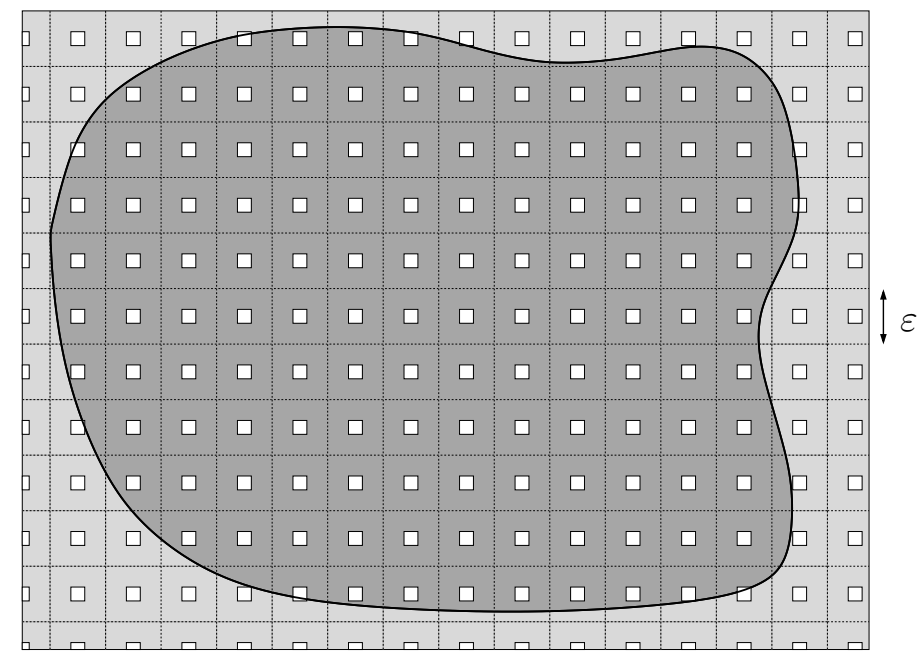

FIGURE 3. In dark grey the stiff matrix $\Omega \cap \varepsilon P$.

for some $c_{3}>0$. In particular, for $i=1,2$, we have that $g_{0}\left(0^{+}, e_{i}\right)=3 / 4$ and $g_{0}\left(t, e_{i}\right)=1$ for $t$ larger than some $t_{0}>0$.

The following remarks are in order.

Remark 3. The homogenisation formula for $\hat{g}$ provided in [5, Theorem 4] gives $\hat{g}\left(e_{1}\right)=\hat{g}\left(e_{2}\right)=$ $3 / 4$ when computed for the micro-geometry $\varepsilon P$ as in Theorem 2. Then, since

$$
\hat{g}(\nu) \leq g_{0}(t, \nu) \quad \text { for every } t>0 \text { and } \nu \in \mathbb{S}^{1} \text {, }
$$

in view of Theorem 2 we may deduce that, for $i=1,2, \hat{g}\left(e_{i}\right)$ is a sharp lower bound for $g_{0}\left(t, e_{i}\right)$ whenever $t \rightarrow 0^{+}$.

Remark 4. In this remark we briefly discuss a few further scalings. To this end let

$$
\begin{aligned}
F_{\varepsilon}^{\alpha, \beta}(u)= & \int_{\Omega \cap \varepsilon P}|\nabla u|^{2} \mathrm{~d} x+\varepsilon^{\alpha} \int_{\Omega \backslash \varepsilon P}|\nabla u|^{2} \mathrm{~d} x \\
& +\mathcal{H}^{1}\left((\Omega \cap \varepsilon P) \cap S_{u}\right)+\varepsilon^{\beta} \mathcal{H}^{1}\left((\Omega \backslash \varepsilon P) \cap S_{u}\right),
\end{aligned}
$$

where $\alpha, \beta>0$ and $\Omega, P$ are chosen as in Theorem 2. The free-discontinuity functionals in (2.9) are a particular case of those analysed by Braides and Solci in [13]. Then, thanks to [13, Theorem 3] it is possible to deduce that for every $\alpha, \beta>0\left(F_{\varepsilon}^{\alpha, \beta}\right) \Gamma$-converges to $\hat{F}$, where $\hat{F}$ is as in (2.1). This implies, in particular, that a limit of cohesive type cannot be obtained if in our model also the toughness of the soft inclusions vanishes as $\varepsilon \rightarrow 0$.

Choosing in (2.9) $\alpha=1$, we end up with the sequence

$$
\begin{aligned}
F_{\varepsilon}^{\beta}(u)= & \int_{\Omega \cap \varepsilon P}|\nabla u|^{2} \mathrm{~d} x+\varepsilon \int_{\Omega \backslash \varepsilon P}|\nabla u|^{2} \mathrm{~d} x \\
& +\mathcal{H}^{1}\left((\Omega \cap \varepsilon P) \cap S_{u}\right)+\varepsilon^{\beta} \mathcal{H}^{1}\left((\Omega \backslash \varepsilon P) \cap S_{u}\right) .
\end{aligned}
$$

Then, our functionals $F_{\varepsilon}$ can be viewed as a "limit" case of $F_{\varepsilon}^{\beta}$ when we let $\beta \rightarrow 0^{+}$. From $[13$, Theorem 3] we know that for every fixed $\beta>0$ the $\Gamma$-limit of $F_{\varepsilon}^{\beta}$ is $\hat{F}$. On the other hand, in Theorem 2 we prove that when $\beta=0$ the $\Gamma$-limit of $F_{\varepsilon}=F_{\varepsilon}^{\beta=0}$ depends on $[u]$ in a nontrivial 
way. This fact can be then interpreted as a sort of non-uniformity (or a lack of continuity) of the $\Gamma$-limit with respect to the exponent $\beta$.

We finally notice that for $\beta=0$ and $\alpha>1$ (which in our notation corresponds to choosing $\left.\delta_{\varepsilon} \ll \varepsilon\right)$ the $\Gamma$-limit is again $\hat{F}$, as it can be seen arguing as in Section 4. Moreover, a heuristic argument also shows that for $\alpha<1$ (or equivalently $\delta_{\varepsilon} \gg \varepsilon$ ) the volume energy density in the $\Gamma$-limit does not change being always equal to $\hat{f}$, while the surface energy density does not depend on the crack-opening and is identically equal to one. Hence in our case $\delta_{\varepsilon}=\varepsilon$ is the only choice leading to a cohesive limit effect. Indeed, at this scaling, bulk and surface energies of the soft part are comparable at a mesoscopic level.

Remark 5. The choice $n=2$ has been only made for the sake of clarity as it simplifies the exposition in the proof of Theorem 2. In fact, Theorem 2 can be implemented in higher dimensions still choosing $\delta_{\varepsilon}=\varepsilon$ and considering the micro-geometry corresponding to

$$
P:=\mathbb{R}^{n} \backslash \bigcup_{i \in \mathbb{Z}^{n}} \bar{Q}_{\frac{1}{4}}+i .
$$

\section{3. $\Gamma$-CONVERGENCE AND INTEGRAL REPRESENTATION}

This section is devoted to the proof of the $\Gamma$-convergence of the functionals $F_{\varepsilon}$.

In order to prove Theorem 1 we use the well-known localisation method of $\Gamma$-convergence (for which we refer the reader to [17, Chapters 14-20]) in combination with the integral-representation result [7, Theorem 1]. To this end, we start introducing the localised functionals as below. We denote by $\mathcal{A}(\Omega)$ the class of all open subsets of $\Omega$ and for every pair $(u, U) \in L^{1}(\Omega) \times \mathcal{A}(\Omega)$ we set

$$
F_{\varepsilon}(u, U):= \begin{cases}\int_{U \cap \varepsilon P}|\nabla u|^{2} \mathrm{~d} x+\int_{U \backslash \varepsilon P}|\nabla u|^{2} \mathrm{~d} x+\mathcal{H}^{n-1}\left(S_{u} \cap U\right) & \text { if } u \in S B V^{2}(U), \\ +\infty & \text { otherwise in } L^{1}(U) .\end{cases}
$$

Moreover, it is also convenient to introduce the following notation:

$$
M S(u, U):= \begin{cases}\int_{U}|\nabla u|^{2} \mathrm{~d} x+\mathcal{H}^{n-1}\left(S_{u} \cap U\right) & \text { if } u \in G S B V^{2}(U), \\ +\infty & \text { otherwise in } L^{1}(U) .\end{cases}
$$

We notice that the functionals $F_{\varepsilon}: L^{1}(\Omega) \times \mathcal{A}(\Omega) \rightarrow[0,+\infty]$ enjoy the following properties: for every $\varepsilon>0, u \in L^{1}(\Omega)$, and $U \in \mathcal{A}(\Omega)$,

$F_{\varepsilon}$ is increasing: $F_{\varepsilon}(u, V) \leq F_{\varepsilon}(u, U)$ for every $V \in \mathcal{A}(\Omega): V \subset U$,

$F_{\varepsilon}$ is local: $F_{\varepsilon}(u, U)=F_{\varepsilon}(v, U)$ for every $v \in L^{1}(\Omega): u=v \mathcal{L}^{n}$-a.e. in $U$,

$F_{\varepsilon}$ decreases by truncation: $F_{\varepsilon}((u \wedge M) \vee(-M), U) \leq F_{\varepsilon}(u, U)$ for $M>0$.

Moreover $F_{\varepsilon}$ is periodic; i.e., for $y_{\varepsilon}:=\left\lfloor\frac{y}{\varepsilon}\right\rfloor \varepsilon$ (here the integer part is meant component-wise)

$$
\begin{gathered}
F_{\varepsilon}\left(u\left(\cdot-y_{\varepsilon}\right), U+y_{\varepsilon}\right)=F_{\varepsilon}(u, U) \text { for all } y \in \mathbb{R}^{n} \text { such that } U+y \subset \subset \Omega \\
\text { and sufficiently small } \varepsilon,
\end{gathered}
$$

and invariant by translations in $u$; i.e.,

$$
F_{\varepsilon}(u+s, U)=F_{\varepsilon}(u, U) \text { for every } s \in \mathbb{R} .
$$


Now let $\left(\varepsilon_{k}\right)$ be a vanishing sequence of strictly positive numbers and define the functionals $F^{\prime}, F^{\prime \prime}: L^{1}(\Omega) \times \mathcal{A}(\Omega) \rightarrow[0,+\infty]$ as

$$
F^{\prime}(\cdot, U):=\Gamma_{k \rightarrow+\infty}^{-\liminf _{n}} F_{\varepsilon_{k}}(\cdot, U) \quad \text { and } \quad F^{\prime \prime}(\cdot, U):=\underset{k \rightarrow+\infty}{-\limsup } F_{\varepsilon_{k}}(\cdot, U),
$$

for every $U \in \mathcal{A}(\Omega)$. The functionals $F^{\prime}, F^{\prime \prime}$ are lower semicontinuous [17, Proposition 6.8] and inherit the properties in (3.2). Specifically, they are increasing [17, Proposition 6.7], local [17, Proposition 16.15], and it is immediate to show that they decrease by truncation. Moreover, in view of [5, Theorem 4], (2.4), and (2.5) we may deduce that the domain of $F^{\prime}$ and $F^{\prime \prime}$ is $G S B V^{2}(\Omega)$ and that for every $(u, U) \in G S B V^{2}(\Omega) \times \mathcal{A}(\Omega)$ the following estimate holds:

$$
\min \left\{c_{1}, c_{2}\right\} M S(u, U) \leq F^{\prime}(u, U) \leq F^{\prime \prime}(u, U) \leq M S(u, U) .
$$

Now fix $\varepsilon>0$ and $u \in G S B V^{2}(\Omega)$; clearly $F_{\varepsilon}(u, \cdot)$ is the restriction to $\mathcal{A}(\Omega)$ of a Radon measure, and therefore $F_{\varepsilon}(u, \cdot)$ is in particular inner regular. On the other hand, $F^{\prime}(u, \cdot), F^{\prime \prime}(u, \cdot)$ are in general not inner regular, hence we also consider their inner regular envelope; i.e., the two functionals $F_{-}^{\prime}, F_{-}^{\prime \prime}: L^{1}(\Omega) \times \mathcal{A}(\Omega) \rightarrow[0,+\infty]$ defined as

$$
F_{-}^{\prime}(u, U):=\sup \left\{F^{\prime}(u, V): V \subset \subset U, V \in \mathcal{A}(\Omega)\right\}
$$

and

$$
F_{-}^{\prime \prime}(u, U):=\sup \left\{F^{\prime \prime}(u, V): V \subset \subset U, V \in \mathcal{A}(\Omega)\right\} .
$$

We notice that $F_{-}^{\prime}$ and $F_{-}^{\prime \prime}$ are both increasing, lower semicontinuous [17, Remark 15.10], and local [17, Remark 15.25]. Moreover, invoking [17, Theorem 16.9] we can find a subsequence $\left(\varepsilon_{k}\right)$ converging to zero such that the corresponding functionals $F^{\prime}$ and $F^{\prime \prime}$ satisfy

$$
F_{-}^{\prime}=F_{-}^{\prime \prime}=: F
$$

Next, appealing to $\left[17\right.$, Proposition 18.6] we show that on $G S B V^{2}(\Omega)$ we actually have $F=$ $F^{\prime}=F^{\prime \prime}$. A preliminary result needed in this direction is the so-called fundamental estimate. The following variant of the fundamental estimate can be derived from the more general [11, Proposition 3.1]. For the reader's convenience we give here a simplified proof relative to our specific case.

Lemma 1 (Fundamental estimate). For every $\eta>0$ and for every $U^{\prime}, U^{\prime \prime}, V \in \mathcal{A}(\Omega)$, with $U^{\prime} \subset \subset U^{\prime \prime}$, there exists a constant $M(\eta)>0$ satisfying the following property: for every $\varepsilon>0$, for every $u \in L^{1}(\Omega)$ with $u \in S B V^{2}\left(U^{\prime \prime}\right)$, and for every $v \in L^{1}(\Omega)$ with $v \in S B V^{2}(V)$, there exists a function $\varphi \in C_{0}^{\infty}(\Omega)$ with $\varphi=1$ in a neighbourhood of $U^{\prime}$, $\operatorname{spt} \varphi \subset U^{\prime \prime}$ and $0 \leq \varphi \leq 1$ such that

$$
F_{\varepsilon}\left(\varphi u+(1-\varphi) v, U^{\prime} \cup V\right) \leq(1+\eta)\left(F_{\varepsilon}\left(u, U^{\prime \prime}\right)+F_{\varepsilon}(u, V)\right)+M(\eta)\|u-v\|_{L^{2}(S)}^{2},
$$

with $S:=\left(U^{\prime \prime} \backslash U^{\prime}\right) \cap V$.

Proof. Let $\eta>0, U^{\prime}, U^{\prime \prime}, V \in \mathcal{A}(\Omega)$ be fixed as in the statement and let $\varphi$ be a function in $C_{0}^{\infty}(\Omega)$ with $0 \leq \varphi \leq 1, \operatorname{spt} \varphi \subset U^{\prime \prime}$ and $\varphi=1$ in a neighbourhood of $U^{\prime}$.

Let $u$ and $v$ be two functions as in the statement and let $w:=\varphi u+(1-\varphi) v$; clearly, $w \in S B V^{2}\left(U^{\prime} \cap V\right)$. Then,

$$
F_{\varepsilon}\left(w, U^{\prime} \cup V\right)=F_{\varepsilon}\left(u, U^{\prime}\right)+F_{\varepsilon}^{*}\left(v, V \backslash U^{\prime \prime}\right)+F_{\varepsilon}^{*}\left(w,\left(U^{\prime \prime} \backslash U^{\prime}\right) \cap V\right),
$$

where for fixed $u \in L^{1}(\Omega), F_{\varepsilon}^{*}(u, \cdot)$ denotes the measure which extends $F_{\varepsilon}(u, \cdot)$ to the $\sigma$-algebra $\mathcal{B}(\Omega)$ of Borel subsets of $\Omega$ in the usual way; i.e.,

$$
F_{\varepsilon}^{*}(u, B):=\inf \left\{F_{\varepsilon}(u, U): U \in \mathcal{A}(\Omega), B \subset U\right\} .
$$


We now estimate the last term in the right-hand side of (3.7). To this end set $S:=\left(U^{\prime \prime} \backslash U^{\prime}\right) \cap V$. For any fixed $\eta \in(0,1)$ we have

$$
\begin{aligned}
F_{\varepsilon}^{*}(w, S) \leq & \int_{S \cap \varepsilon P}\left|(1-\eta) \frac{\varphi \nabla u+(1-\varphi) \nabla v}{1-\eta}+\eta \frac{\nabla \varphi(u-v)}{\eta}\right|^{2} \mathrm{~d} x \\
& +\delta_{\varepsilon} \int_{S \backslash \varepsilon P}\left|(1-\eta) \frac{\varphi \nabla u+(1-\varphi) \nabla v}{1-\eta}+\eta \frac{\nabla \varphi(u-v)}{\eta}\right|^{2} \mathrm{~d} x \\
& +\mathcal{H}^{n-1}\left(S_{u} \cap S\right)+\mathcal{H}^{n-1}\left(S_{v} \cap S\right) \\
\leq & \frac{1}{1-\eta}\left(\int_{S \cap \varepsilon P}|\nabla u|^{2} \mathrm{~d} x+\int_{S \cap \varepsilon P}|\nabla v|^{2} \mathrm{~d} x\right) \\
& +\frac{\delta_{\varepsilon}}{1-\eta}\left(\int_{S \backslash \varepsilon P}|\nabla u|^{2} \mathrm{~d} x+\int_{S \backslash \varepsilon P}|\nabla v|^{2} \mathrm{~d} x\right) \\
& +\frac{1}{\eta} \int_{S}|\nabla \varphi|^{2}|u-v|^{2} \mathrm{~d} x+\mathcal{H}^{n-1}\left(S_{u} \cap S\right)+\mathcal{H}^{n-1}\left(S_{v} \cap S\right) \\
\leq & \frac{1}{1-\eta}\left(F_{\varepsilon}^{*}(u, S)+F_{\varepsilon}^{*}(v, S)\right)+\frac{1}{\eta} \int_{S}|\nabla \varphi|^{2}|u-v|^{2} \mathrm{~d} x .
\end{aligned}
$$

Finally, setting $M:=\|\nabla \varphi\|_{L^{\infty}}$ and combining (3.7) and (3.8) give

$$
F_{\varepsilon}\left(w, U^{\prime} \cup V\right) \leq \frac{1}{1-\eta}\left(F_{\varepsilon}\left(u, U^{\prime \prime}\right)+F_{\varepsilon}(v, V)\right)+\frac{M}{\eta}\|u-v\|_{L^{2}(S)}^{2},
$$

and hence the thesis.

We are now ready to state and prove a compactness result by $\Gamma$-convergence. We notice that in the proof of Theorem 3 below the only difference from the general approach developed in [17, Chapter 18] is that in our case the rest in the fundamental estimate is small when computed along sequences converging in $L^{2}(\Omega)$ (see Lemma 1) while we are interested in the $\Gamma$-convergence of $F_{\varepsilon}$ with respect to the $L^{1}(\Omega)$-topology.

Theorem 3 (Compactness by $\Gamma$-convergence). Let $F$ be as in (3.6). Then

(a) (measure property) for every $u \in G S B V^{2}(\Omega)$ the set function $F(u, \cdot)$ is the restriction to $\mathcal{A}(\Omega)$ of a Radon measure on $\Omega$;

(b) (compactness) for every $U \in \mathcal{A}(\Omega)$

$$
F(\cdot, U)=F^{\prime}(\cdot, U)=F^{\prime \prime}(\cdot, U) \quad \text { on } G S B V^{2}(\Omega) ;
$$

(c) (translational invariance in $x$ ) for every $u \in L^{1}(\Omega)$ and $U \in \mathcal{A}(\Omega)$

$$
F(u(\cdot-y), U+y)=F(u, U) \text { for all } y \in \mathbb{R}^{n} \text { such that } U+y \subset \subset \Omega ;
$$

(d) (translational invariance in $u$ ) for every $u \in L^{1}(\Omega)$ and $U \in \mathcal{A}(\Omega)$

$$
F(u+s, U)=F(u, U) \text { for all } s \in \mathbb{R} .
$$

Proof. The proof of (a) relies on the measure-property criterion of De Giorgi and Letta [17, Theorem 14.23]. The only delicate point here is to show that $F$ is subadditive. This can be handled first appealing to a standard truncation argument and to the the fact that on $S B V^{2}(\Omega) \cap L^{\infty}(\Omega)$ there is an equivalent sequential charactherization of (3.6) (see also [17, Proposition 16.4 and Remark 16.5]) and then arguing as in e.g. [21, Theorem 3.4]. 
The proof of (b) readily follows from (3.6) once we prove that, for every $u \in G S B V^{2}(\Omega)$, $F^{\prime \prime}(u, \cdot)$ is inner regular. Indeed, by definition of inner regular envelope and by monotonicity we always have

$$
F=F_{-}^{\prime \prime}=F_{-}^{\prime} \leq F^{\prime} \leq F^{\prime \prime},
$$

hence if we show that $F^{\prime \prime} \leq F_{-}^{\prime \prime}$ (which is equivalent to the inner regularity of $F^{\prime \prime}$ ), from (3.9) we may conclude that $F=F^{\prime}=F^{\prime \prime}$ and hence (b).

Appealing to Lemma 1 and to the upper bound in (3.5), we now prove that for every fixed $u \in$ $G S B V^{2}(\Omega)$ the set function $F^{\prime \prime}(u, \cdot)$ is inner regular on $\mathcal{A}(\Omega)$. To this end, let $u \in G S B V^{2}(\Omega)$ and fix $W \in \mathcal{A}(\Omega)$. Since $M S(u, \cdot)$ is a Radon measure, for every $\eta>0$ there exists a compact set $K \subset W$ such that $M S(u, W \backslash K)<\eta$.

Choose $U, U^{\prime} \in \mathcal{A}(\Omega)$ satisfying $K \subset U^{\prime} \subset \subset U \subset \subset W$ and set $V:=W \backslash K$. Recalling that $F^{\prime \prime}$ is increasing, Lemma 1 easily yields

$$
F^{\prime \prime}(u, W) \leq F^{\prime \prime}\left(u, U^{\prime} \cup V\right) \leq F^{\prime \prime}(u, U)+F^{\prime \prime}(u, V)=F^{\prime \prime}(u, U)+F^{\prime \prime}(u, W \backslash K) .
$$

Moreover, by the definition of $F_{-}^{\prime \prime}$ and in view of the bound $F^{\prime \prime} \leq M S$ we have

$$
F^{\prime \prime}(u, W) \leq F_{-}^{\prime \prime}(u, W)+M S(u, W \backslash K) \leq F_{-}^{\prime \prime}(u, W)+\eta .
$$

Hence by the arbitrariness of $\eta>0$ we get

$$
F^{\prime \prime}(u, W) \leq F_{-}^{\prime \prime}(u, W) \quad \text { for every } W \in \mathcal{A}(\Omega),
$$

and thus the inner regularity of $F^{\prime \prime}(u, \cdot)$ for $u \in G S B V^{2}(\Omega)$.

Finally, by virtue of (3.3) and (3.4), the proof of (c) and (d) follows by standard arguments (see e.g. [11, Lemma 3.7])

On account of the compactness result Theorem 3 we are now ready to accomplish the proof of Theorem 1, that is to represent the $\Gamma$-limit $F$ in an integral form. This last step will be accomplished by means of a standard truncation argument and then appealing to the representation result [7, Theorem 1]. For the reader's convenience, below we rephrase [7, Theorem 1] adapting it to our specific setting.

Theorem 4. Let $G: S B V^{2}(\Omega) \times \mathcal{A}(\Omega) \rightarrow[0,+\infty]$ be a functional satisfying for every $(u, U) \in$ $S B V^{2}(\Omega) \times \mathcal{A}(\Omega)$ the following conditions:

(i) $G(\cdot, U)$ is local;

(ii) $G(\cdot, U)$ is $L^{1}(\Omega)$-lower semicontinuous;

(iii) $G(u, \cdot)$ is the restriction to $\mathcal{A}(\Omega)$ of a Radon measure;

(iv) $G(\cdot, U)$ is invariant under translation in $x$ and in $u$;

(v) there exists $C>0$ such that

$$
\frac{1}{C} M S(u, U) \leq G(u, U) \leq C(M S(u, U)+1) .
$$

Then, there exist Borel functions $f_{0}: \mathbb{R}^{n} \rightarrow[0,+\infty)$ and $g_{0}: \mathbb{R} \times S^{n-1} \rightarrow[0,+\infty)$ such that

$$
G(u, U)=\int_{U} f_{0}(\nabla u) \mathrm{d} x+\int_{S_{u} \cap U} g_{0}\left([u], \nu_{u}\right) \mathrm{d} \mathcal{H}^{n-1}
$$

for every pair $(u, U) \in S B V^{2}(\Omega) \times \mathcal{A}(\Omega)$.

Moreover, $g_{0}(t, \nu)=g_{0}(-t,-\nu)$ for every $t \in \mathbb{R}$, and $\nu \in S^{n-1}$. 
Proof. The proof follows from [7, Theorem 1] by means of a perturbation argument. To this end let $\sigma>0$ and for every $(u, U) \in S B V^{2}(\Omega) \times \mathcal{A}(\Omega)$ define the functionals

$$
G_{\sigma}(u, U):=G(u, U)+\sigma \int_{S_{u} \cap U}(1+|[u]|) \mathrm{d} \mathcal{H}^{n-1} .
$$

Clearly

$$
\begin{aligned}
& G_{\sigma}(u, U) \geq \frac{1}{C_{\sigma}}\left(M S(u, U)+\int_{S_{u} \cap U}|[u]| \mathrm{d} \mathcal{H}^{n-1}\right), \\
& G_{\sigma}(u, U) \leq C_{\sigma}\left(M S(u, U)+\int_{S_{u} \cap U}|[u]| \mathrm{d} \mathcal{H}^{n-1}+1\right)
\end{aligned}
$$

for $C_{\sigma}>0$, thus $G_{\sigma}$ satisfies the growth conditions in [7, Theorem 1]. Moreover, it is immediate to check that $G_{\sigma}$ satisfies the other hypotheses of [7, Theorem 1] (the lower semicontinuity being a consequence of $[1$, Theorem 3.7]). Then, we deduce the existence of two Borel functions $f_{\sigma}: \mathbb{R}^{n} \rightarrow[0,+\infty)$ and $g_{\sigma}: \mathbb{R} \times \mathbb{S}^{n-1} \rightarrow[0,+\infty)$ such that

$$
G_{\sigma}(u, U)=\int_{U} f_{\sigma}(\nabla u) \mathrm{d} x+\int_{S_{u} \cap U} g_{\sigma}\left([u], \nu_{u}\right) \mathrm{d} \mathcal{H}^{n-1}
$$

for every $(u, U) \in S B V^{2}(\Omega) \times \mathcal{A}(\Omega)$.

By construction the two families of functions $\left(f_{\sigma}\right)$ and $\left(g_{\sigma}\right)$ are decreasing as $\sigma$ decreases (cf. equations (1)-(3), [7, Theorem 1]). Hence, setting $f_{0}:=\lim _{\sigma \rightarrow 0^{+}} f_{\sigma}$ and $g_{0}:=\lim _{\sigma \rightarrow 0^{+}} g_{\sigma}$, by the pointwise convergence of $\left(G_{\sigma}\right)$ to $G$ and the Monotone Convergence Theorem we deduce

$$
G(u, U)=\int_{U} f_{0}(\nabla u) \mathrm{d} x+\int_{S_{u} \cap U} g_{0}\left([u], \nu_{u}\right) \mathrm{d} \mathcal{H}^{n-1}
$$

for every $(u, U) \in S B V^{2}(\Omega) \times \mathcal{A}(\Omega)$, and thus the thesis.

We are now in a position to prove the $\Gamma$-convergence result Theorem 1.

Proof of Theorem 1. Let $F$ be as in (3.6) and let $\left(\varepsilon_{k}\right)$ be the corresponding vanishing subsequence. Then, Theorem 3 ensures that

$$
F(u, U):=\Gamma_{k \rightarrow+\infty} \lim _{\varepsilon_{k}}(u, U)
$$

for every $(u, U) \in G S B V^{2}(\Omega) \times \mathcal{A}(\Omega)$ and hence, in particular, that $F(\cdot, U)$ is $L^{1}(\Omega)$-lower semicontinuous on $G S B V^{2}(\Omega)$. Moreover, again appealing to Theorem 3 and to the bound (3.5) ensures that $F$ fulfils the set of hypotheses of Theorem 4 . Therefore we deduce the existence of two Borel functions $f_{0}: \mathbb{R}^{n} \rightarrow[0,+\infty)$ and $g_{0}: \mathbb{R} \times S^{n-1} \rightarrow[0,+\infty)$ such that

$$
F(u, U)=\int_{U} f_{0}(\nabla u) \mathrm{d} x+\int_{S_{u} \cap U} g_{0}\left([u], \nu_{u}\right) \mathrm{d} \mathcal{H}^{n-1},
$$

for every $(u, U) \in S B V^{2}(\Omega) \times \mathcal{A}(\Omega)$.

Let $y \in \mathbb{R}^{n}, r>0$, and $\nu \in \mathbb{S}^{n-1}$. We denote by $Q_{r}^{\nu}(y)$ the open cube of centre $y$, side-length $r>0$, and one face orthogonal to $\nu$. Now fix $y \in \Omega$ and let $r>0$ be such that $Q_{r}^{\nu}(y) \subset \Omega$; then the bounds $c_{1}|\xi|^{2} \leq f_{0}(\xi) \leq|\xi|^{2}$ and $c_{2} \leq g_{0}(t, \nu) \leq 1$ readily follow from (2.1), (2.2), (the localised version of) $(2.4)$, and (3.10) by testing $F\left(\cdot, Q_{r}^{\nu}(y)\right)$ on

$$
u_{\xi}(x)=\xi \cdot x \quad \text { and } \quad u_{t}^{\nu}(x)= \begin{cases}t & \text { if }(x-y) \cdot \nu>0 \\ 0 & \text { if }(x-y) \cdot \nu \leq 0\end{cases}
$$

respectively. 
Moreover, it is immediate to prove that $g_{0}(\cdot, \nu)$ is nondecreasing in $(0,+\infty)$ for every fixed $\nu \in \mathbb{S}^{n-1}$ (see e.g. [21, Proof of Theorem 2.2]) while the symmetry property directly follows from [7, Theorem 1]. Finally, the left-continuity of $g_{0}(\cdot, \nu)$ in $(0,+\infty)$ is a consequence of its monotonicity and of the lower semicontinuity of $F$.

We now show that the representation formula for $F$ holds on the whole $G S B V^{2}(\Omega)$. To this end, for $(u, U) \in G S B V^{2}(\Omega) \times \mathcal{A}(\Omega)$ set

$$
\widetilde{F}(u, U):=\int_{U} f_{0}(\nabla u) \mathrm{d} x+\int_{S_{u} \cap U} g_{0}\left([u], \nu_{u}\right) \mathrm{d} \mathcal{H}^{n-1},
$$

and for every $M>0$ let $u^{M}:=(u \wedge M) \vee(-M)$. Notice that $\widetilde{F}$ decreases by truncation and that it is $L^{1}(\Omega)$-lower semicontinuous on $G S B V^{2}(\Omega)$. Then by the $L^{1}(\Omega)$-lower semicontinuity of $F$ on $G S B V^{2}(\Omega)$ and by (3.10) we have

$$
F(u, U) \leq \liminf _{M \rightarrow+\infty} F\left(u^{M}, U\right)=\liminf _{M \rightarrow+\infty} \widetilde{F}\left(u^{M}, U\right) \leq \widetilde{F}(u, U) .
$$

On the other hand, since $F$ decreases by truncation we may also deduce

$$
\widetilde{F}(u, U) \leq \liminf _{M \rightarrow+\infty} \widetilde{F}\left(u^{M}, U\right)=\liminf _{M \rightarrow+\infty} F\left(u^{M}, U\right) \leq F(u, U) .
$$

Hence gathering (3.11) and (3.12) gives the desired representation result on $G S B V^{2}(\Omega)$. Then, choosing $U=\Omega$ yields the $\Gamma$-convergence result.

To conclude the proof it only remains to show that $f_{0}=\hat{f}$. To this end, for any fixed $\delta \in(0,1)$ consider the functionals $F_{\varepsilon}^{\delta}: L^{1}(\Omega) \rightarrow[0,+\infty]$ given by

$$
F_{\varepsilon}^{\delta}(u):= \begin{cases}\int_{\Omega \cap \varepsilon P}|\nabla u|^{2} \mathrm{~d} x+\delta \int_{\Omega \backslash \varepsilon P}|\nabla u|^{2} \mathrm{~d} x & \text { if } u \in H^{1}(\Omega), \\ +\infty & \text { otherwise in } L^{1}(\Omega) .\end{cases}
$$

Then, $\left(F_{\varepsilon}^{\delta}\right) \Gamma$-converges to the quadratic form

$$
F^{\delta}(u):= \begin{cases}\int_{\Omega} f^{\delta}(\nabla u) \mathrm{d} x & \text { if } u \in H^{1}(\Omega), \\ +\infty & \text { otherwise in } L^{1}(\Omega),\end{cases}
$$

where $f^{\delta}: \mathbb{R}^{n} \rightarrow[0,+\infty)$ is defined as

$$
f^{\delta}(\xi)=\min \left\{\int_{Q}\left(\chi_{Q \cap P}(x)+\delta \chi_{Q \backslash P}(x)\right)|\xi+\nabla w|^{2} \mathrm{~d} x: w \in H_{\mathrm{per}}^{1}(Q)\right\},
$$

for every $\xi \in \mathbb{R}^{n}$ (see $\left[9\right.$, Subsection 5.2]). For sufficiently small $\varepsilon>0$ we have $\hat{F}_{\varepsilon} \leq F_{\varepsilon} \leq F_{\varepsilon}^{\delta}$ and therefore $\hat{f} \leq f_{0} \leq f^{\delta}$. We show that

$$
\lim _{\delta \rightarrow 0^{+}} f^{\delta}(\xi)=\hat{f}(\xi)
$$

for every $\xi \in \mathbb{R}^{n}$. To this end, we first assume that $P$ is Lipschitz so that

$$
\hat{f}(\xi)=\inf \left\{\int_{Q \cap P}|\xi+\nabla w|^{2} \mathrm{~d} x: w \in H_{\mathrm{per}}^{1}(Q)\right\} .
$$

Fix $\xi \in \mathbb{R}^{n}$; for any given $\eta>0$, let $w^{\eta} \in H_{\text {per }}^{1}(Q)$ be such that

$$
\int_{Q \cap P}\left|\xi+\nabla w^{\eta}\right|^{2} \mathrm{~d} x \leq \hat{f}(\xi)+\eta
$$


Then

$$
f^{\delta}(\xi) \leq \int_{Q}\left(\chi_{Q \cap P}(x)+\delta \chi_{Q \backslash P}(x)\right)\left|\xi+\nabla w^{\eta}\right|^{2} \mathrm{~d} x \leq \hat{f}(\xi)+\eta+\delta \int_{Q \backslash P}\left|\xi+\nabla w^{\eta}\right|^{2} \mathrm{~d} x .
$$

Thus (3.14) follows by letting $\delta$ tend to zero and by the arbitrariness of $\eta>0$.

Finally, if $P$ is not Lipschitz, the fact that $f_{0}=\hat{f}$ can be recovered as in [5, equation (44)] by approximating $P$ with an increasing sequence of Lipschitz open sets $\left(P_{m}\right)$ invading $P$.

\section{Dependence on the CRACK-Opening}

In the present section we prove the main result of this paper, namely Theorem 2 . To this end, we need several ingredients which are collected in the following lemmas.

Henceforth, without loss of generality, we restrict ourselves to the case $\Omega=Q$, cf. (4.2).

Lemma 2. Let $\left(U_{k}\right)$ be a sequence of Borel subsets of $Q$ such that $\chi_{U_{k}} \rightarrow \theta$ weakly* in $L^{\infty}(Q)$, with $\theta \in(0,1]$. Let $\left(u_{k}\right),\left(v_{k}\right)$ be two sequences in $L^{1}(Q)$ such that $u_{k}=v_{k} \mathcal{L}^{2}$-a.e. in $U_{k}$. Then, if $u_{k} \rightarrow u$ and $v_{k} \rightarrow v$ in $L^{1}(Q)$, we have $u=v \mathcal{L}^{2}$-a.e. in $Q$.

Proof. For every fixed $k \in \mathbb{N},\left(u_{k}-v_{k}\right) \chi_{U_{k}}=0 \quad \mathcal{L}^{2}$-a.e. in $Q$. On the other hand $\left(u_{k}-v_{k}\right) \chi_{U_{k}} \rightarrow$ $(u-v) \theta$ weakly in $L^{1}(Q)$, then by virtue of the positivity of $\theta$ we immediately infer that $u=v$ $\mathcal{L}^{2}$-a.e. in $Q$.

The following lemma allows us to replace a converging sequence $\left(u_{k}\right)$ by a sequence of functions $v_{k}$, periodic and symmetric in the first variable, still converging to the same limit, without essentially increasing the energy $F_{\varepsilon}$.

Lemma 3. Set $u_{t}:=t \chi_{Q \cap\left\{x_{2}>0\right\}}$ and let $\left(u_{k}\right) \subset L^{1}(Q)$ be such that $u_{k} \rightarrow u_{t}$ in $L^{1}(Q)$. Assume that $\left(\varepsilon_{k}^{-1}\right)$ is a sequence of odd integers (so that $Q$ is union of periodicity cells). Then, for any fixed $\eta>0$ there exists $\left(v_{k}\right) \subset L^{1}(Q), \varepsilon_{k}$-periodic and symmetric in its first variable (i.e., $v_{k}\left(x_{1}, x_{2}\right)=v_{k}\left(x_{1}+\varepsilon_{k}, x_{2}\right)$ and $v_{k}\left(x_{1}, x_{2}\right)=v_{k}\left(-x_{1}, x_{2}\right)$ for every $\left.\left(x_{1}, x_{2}\right) \in Q\right)$ such that $v_{k} \rightarrow u_{t}$ in $L^{1}(Q)$ and

$$
\liminf _{k \rightarrow+\infty} F_{\varepsilon_{k}}\left(u_{k}\right) \geq \liminf _{k \rightarrow+\infty} F_{\varepsilon_{k}}\left(v_{k}\right)-\eta .
$$

Proof. Let $S_{k}^{i}$ be the open strip $\left(i \varepsilon_{k} / 2,(i+1) \varepsilon_{k} / 2\right) \times(-1 / 2,1 / 2)$ and let $\left(\lambda_{k}\right)$ be a sequence such that

$$
\lambda_{k} \rightarrow+\infty \quad \text { and } \sup _{k}\left(\lambda_{k} \int_{Q}\left|u_{k}-u_{t}\right| \mathrm{d} x\right) \leq \eta .
$$

Let $i_{k} \in \mathbb{Z}$ be a solution to

$$
\min \left\{F_{\varepsilon_{k}}\left(u_{k}, S_{k}^{i}\right)+\lambda_{k} \int_{S_{k}^{i}}\left|u_{k}-u_{t}\right| \mathrm{d} x: i=-\varepsilon_{k}^{-1}, \ldots, \varepsilon_{k}^{-1}-1\right\},
$$

where $F_{\varepsilon_{k}}\left(u_{k}, S_{k}^{i}\right)$ is defined as in (3.1). In order to define $v_{k}$ we first extend $\left.u_{k}\right|_{S_{k}}$ to the strip $\left(\left(i_{k}-1\right) \varepsilon_{k} / 2,\left(i_{k}+1\right) \varepsilon_{k} / 2\right) \times(-1 / 2,1 / 2)$ by reflection with respect to $x_{1}=i_{k} \varepsilon_{k} / 2$; we denote by $\tilde{u}_{k}$ such an extension. Then we extend $\tilde{u}_{k}$ by $\varepsilon_{k}$-periodicity in the $x_{1}$-variable to the whole $\mathbb{R} \times(-1 / 2,1 / 2)$. Hence we call $v_{k}$ the latter extension. Then, (4.1) holds true by definition of $v_{k}$, while the penalisation term also ensures that $v_{k} \rightarrow u_{t}$ in $L^{1}(Q)$.

Lemma 5 below represents a key ingredient in the proof of Theorem 2 and is a consequence of the elimination lemma [19, Lemma 0.7], which we recall for the reader's convenience. 


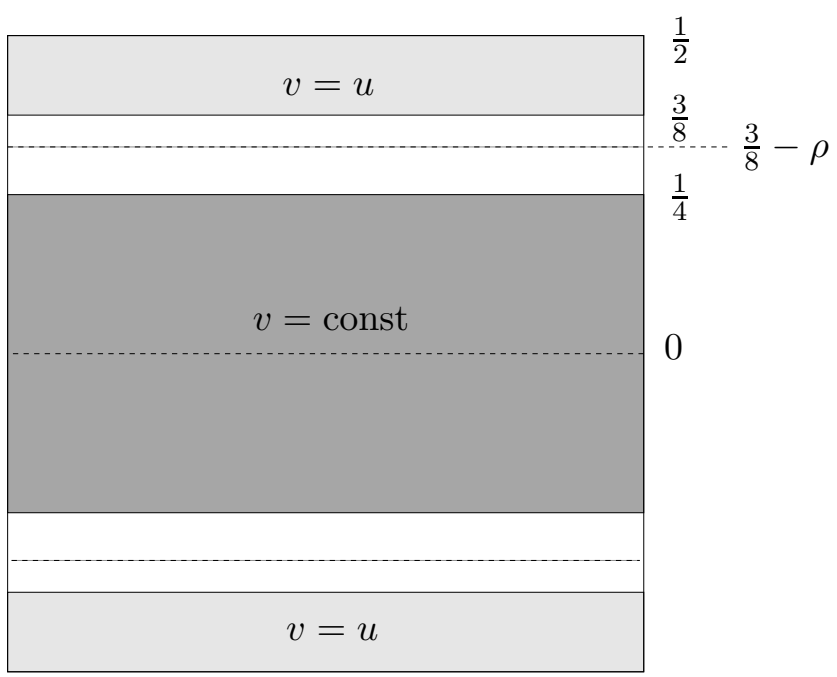

Figure 4. The function $v$ of Lemma 5.

Definition 1. Let $V \subset \mathbb{R}^{n}$ be open. We say that $v \in S B V^{2}(V) \cap L^{\infty}(V)$ is a local minimiser for $M S(\cdot, V)$ if $M S(v, A) \leq M S(w, A)$ for every open set $A \subset \subset V$, whenever $w \in S B V^{2}(V) \cap L^{\infty}(V)$ and $\{w \neq v\} \subset \subset A \subset \subset V$.

Lemma 4 (Elimination property). Let $V \subset \mathbb{R}^{n}$ be open. There exists a dimensional constant $\vartheta=\vartheta(n)>0$, independent of $V$, such that, if $v \in S B V^{2}(V) \cap L^{\infty}(V)$ is a local minimiser for $M S(\cdot, V)$ and $B_{2 \rho}(\bar{x}) \subset V$ is any ball with centre $x_{0} \in V$ and radius $2 \rho$ with

$$
\mathcal{H}^{n-1}\left(S_{v} \cap B_{2 \rho}(\bar{x})\right)<\vartheta(2 \rho)^{n-1},
$$

then $u \in H^{1}\left(B_{\rho}\right)$.

Lemma 5. There exist two constants $\alpha, \beta>0$ with the following property: Let $u \in S B V^{2}(Q) \cap$ $L^{\infty}(Q)$ be symmetric in its first variable and such that $M S(u, U) \leq \beta$, where $U:=(-1 / 2,1 / 2) \times$ $(-3 / 8,3 / 8)$. Then there exists a function $v \in S B V^{2}(Q) \cap L^{\infty}(Q)$ still symmetric and such that

(i) $v$ is constant in $(-1 / 2,1 / 2) \times(-1 / 4,1 / 4)$;

(ii) $v=u$ in $Q \backslash U$;

(iii) $\|v\|_{L^{\infty}(Q)} \leq\|u\|_{L^{\infty}(Q)}$;

(iv) $\alpha \int_{Q}|\nabla v|^{2} \mathrm{~d} x+\mathcal{H}^{1}\left(S_{v} \cap Q\right) \leq M S(u, Q)$.

Proof. We first substitute $u$ with a solution $w$ to the minimisation problem

$$
\min \left\{M S(w, Q): w \in S B V^{2}(Q) \cap L^{\infty}(Q), w=u \text { in } Q \backslash U\right\} .
$$

We clearly have $\|w\|_{L^{\infty}(Q)} \leq\|u\|_{L^{\infty}(Q)}$. Since $u$ is symmetric in $x_{1}$, we can always assume that $w$ inherits the same property. Moreover, we can extend $w$ by reflection to $(-3 / 2,3 / 2) \times(-1 / 2,1 / 2)$ keeping the minimality with respect to all competitors coinciding with $u$ in $(-3 / 2,3 / 2) \times$ $((-1 / 2,1 / 2) \backslash(-3 / 8,3 / 8))$, where $u$ is extended by reflection, too. This allows us to apply the elimination property Lemma 4. More precisely, let $\rho \in(0,1 / 8)$ be fixed and $\beta:=2 \rho \vartheta$, where $\vartheta=\vartheta(2)$ is as in Lemma 4 . If $B_{2 \rho}(\bar{x}) \subset(-3 / 2,3 / 2) \times(-3 / 8,3 / 8)$, then our assumptions imply $\mathcal{H}^{1}\left(S_{w} \cap B_{2 \rho}(\bar{x})\right) \leq \beta$, hence in its turn the elimination property ensures that $w \in H^{1}\left(B_{\rho}(\bar{x})\right)$. By moving $\bar{x}$ in $(-3 / 2,3 / 2) \times[-3 / 8+2 \rho, 3 / 8-2 \rho]$, we infer that $w \in$ $H^{1}((-1 / 2,1 / 2) \times(-3 / 8+\rho, 3 / 8-\rho))$. 


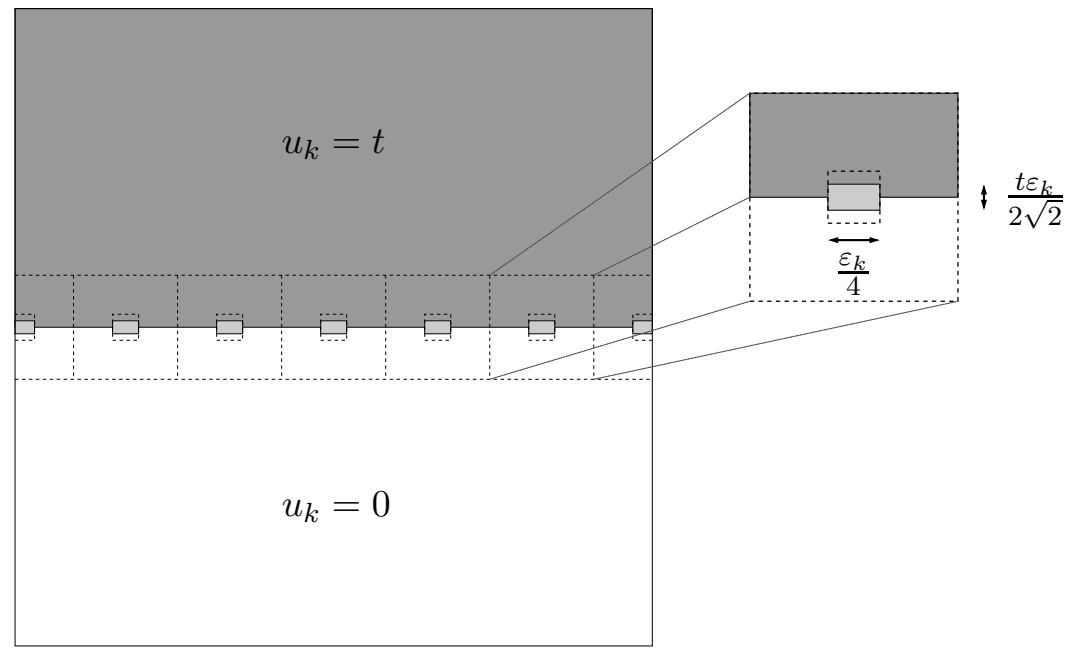

Figure 5. The "bridging" sequence $\left(u_{k}\right)$. The discontinuity set $S_{u_{k}}$ is given by the union of the horizontal interface between the white and the dark grey zone and the vertical part of the boundary of the light grey rectangles. The light grey rectangles (where $u_{k}$ is affine) are contained in the soft inclusions and act as "bridging elements" in the deformed configuration.

The desired function $v$ is finally obtained by means of a standard cut-off argument (see Figure 4). Specifically, let $\varphi$ be a smooth function defined on $Q$ and depending only on the second variable, with $\varphi\left(x_{1}, x_{2}\right)=1$ for $\left|x_{2}\right|>5 / 16$ and $\varphi\left(x_{1}, x_{2}\right)=0$ for $\left|x_{2}\right|<1 / 4$. We define $v:=(w-m) \varphi+m$, where $m$ is the mean value of $w$ on $(-1 / 2,1 / 2) \times(-5 / 16,5 / 16)$. Then (i)-(iii) hold by construction, while (iv) follows by the Poincaré-Wirtinger inequality, recalling that $w$ is a Sobolev function in $(-1 / 2,1 / 2) \times(-5 / 16,5 / 16)$.

Remark 6. It is worth mentioning that the previous lemma can be generalised to any dimension $n$ with some $\beta=\beta(n)$ (compare with Lemma 7 in Section 5 which is a purely two-dimensional argument). Moreover, by using the scaled functions $x \mapsto u(\varepsilon x) / \sqrt{\varepsilon}$, Lemma 5 can be stated in the cube $Q_{\varepsilon}$ up to replacing $\beta$ by $\varepsilon^{n-1} \beta$.

We are now in a position to prove Theorem 2. By using approximate displacements $u_{k}$ mimicking a "bridging effect" in the microstructure (see e.g. Figure 5), we will show that the surface energy density $g_{0}$ depends on the crack-opening in a nontrivial way.

Proof of Theorem 2. We start noticing that in view of the symmetry of the microgeometry the functionals $F_{\varepsilon}$ are invariant under ninety-degree rotations. Then it is immediate to check that $g_{0}\left(t, e_{1}\right)=g_{0}\left(t, e_{2}\right)$ for every $t \geq 0$. Thus it is enough to prove (2.8) when $i=2$. By Theorem 1 we have that

$$
g_{0}\left(t, e_{2}\right)=F\left(u_{t}, Q\right), \quad \text { where } \quad u_{t}:=t \chi_{Q \cap\left\{x_{2}>0\right\}} .
$$

We divide the proof into two main parts.

Estimate from above. By (2.4) we immediately deduce that $g_{0}\left(t, e_{2}\right) \leq 1$ for every $t>0$. Then, to prove that $g_{0}\left(\cdot, e_{2}\right)$ satisfies the estimate from above in $(2.8)$, we need to show that $g_{0}\left(t, e_{2}\right) \leq 3 / 4+\sqrt{2} t$ whenever $t \leq 1 /(4 \sqrt{2})$. To this end, let $t \leq 1 /(4 \sqrt{2})$ and let $R \subset \mathbb{R}^{2}$ be the open rectangle defined as

$$
R:=\left(-\frac{1}{8}, \frac{1}{8}\right) \times\left(-\frac{t}{4 \sqrt{2}}, \frac{t}{4 \sqrt{2}}\right) .
$$


With this choice of $t$ we also have $R \subset Q_{1 / 4}$. Set

$$
R_{k}:=Q \cap \varepsilon_{k} \bigcup_{i \in \mathbb{Z}}(R+(i, 0))
$$

and let $\left(u_{k}\right) \subset S B V^{2}(Q)$ be the sequence of "bridging" functions defined as

$$
u_{k}(x):= \begin{cases}t & \text { if } x \in Q \backslash R_{k} \text { and } x_{2} \geq 0, \\ \frac{t}{2}+\frac{2 \sqrt{2}}{\varepsilon_{k}} x_{2} & \text { if } x \in R_{k}, \\ 0 & \text { if } x \in Q \backslash R_{k} \text { and } x_{2}<0,\end{cases}
$$

(see Figure 5). We clearly have $u_{k} \rightarrow u_{t}$ in $L^{1}(Q)$; moreover

$$
\int_{R_{k}}\left|\nabla u_{k}\right|^{2} \mathrm{~d} x \leq\left(\left\lfloor\frac{1}{\varepsilon_{k}}\right\rfloor+1\right)\left(\frac{t}{\sqrt{2}}\right) \quad \text { and } \quad \mathcal{H}^{1}\left(S_{u_{k}}\right) \leq \varepsilon_{k}\left(\left\lfloor\frac{1}{\varepsilon_{k}}\right\rfloor+1\right)\left(\frac{3}{4}+\frac{t}{\sqrt{2}}\right) .
$$

Thus we readily deduce

$$
g_{0}\left(t, e_{2}\right)=F\left(u_{t}, Q\right) \leq \limsup _{k \rightarrow+\infty} F_{\varepsilon_{k}}\left(u_{k}, Q\right) \leq \frac{3}{4}+\sqrt{2} t,
$$

and hence the estimate from above.

Estimate from below. Let $u_{k} \rightarrow u_{t}$ in $L^{1}(Q)$; we want to prove that

$$
\liminf _{k} F_{\varepsilon_{k}}\left(u_{k}, Q\right) \geq \min \left\{\frac{3}{4}+c_{3} t^{2}, 1\right\}
$$

for some $c_{3}>0$.

The strategy of the proof is to show that the energy essentially concentrates in a horizontal layer of thickness proportional to $\varepsilon_{k}$, giving a contribution controlled from below as in (4.3); this is shown in Step 4 . In order to prove this, we suitably modify $\left(u_{k}\right)$ obtaining a new sequence $\left(w_{k}\right)$ that is Sobolev outside the layer as above and whose gradient has equibounded $L^{2}$-norm in the same layer. The construction of the sequence $\left(w_{k}\right)$ is performed in Steps 1, 2, and 3 and is based on consecutive modifications of $\left(u_{k}\right)$, which have the property of keeping the limit $u_{t}$. Indeed, at each step the sequence remains unchanged in "large" portions of each periodicity cell, so that the convergence to $u_{t}$ is preserved thanks to Lemma 2. Moreover the various modifications of $\left(u_{k}\right)$ are such that their energy changes in a way that can be suitably controlled in terms of $F_{\varepsilon_{k}}\left(u_{k}, Q\right)$.

We start noticing that we may assume

$$
\liminf _{k} F_{\varepsilon_{k}}\left(u_{k}, Q\right) \leq 1,
$$

otherwise there is nothing to prove. Up to an extraction of a subsequence, we can additionally assume that the liminf in (4.4) is actually a limit. In this way we can modify the sequence $\left(u_{k}\right)$, possibly extracting further subsequences, keeping the estimates.

Since $F_{\varepsilon_{k}}$ decreases by truncation, we can also suppose that $0 \leq u_{k} \leq t$ in $Q$. Moreover, we assume that $\varepsilon_{k}^{-1}$ is an odd integer and, in view of Lemma 3, that $u_{k}$ is $\varepsilon_{k}$-periodic and symmetric in its first variable.

We divide the proof into four steps.

Step 1. Appealing to Lemma 5 , in this step we modify $u_{k}$ replacing it by constant values in suitably chosen regions of the domain $Q$.

We decompose $Q$ into squares of side-length $\varepsilon_{k}$ by setting $Q_{\varepsilon_{k}}^{i, j}:=Q_{\varepsilon_{k}}+p_{k}^{i, j}$, where

$$
p_{k}^{i, j}:=\left(-\frac{1}{2}+\left(i-\frac{1}{2}\right) \varepsilon_{k},-\frac{1}{2}+\left(j-\frac{1}{2}\right) \varepsilon_{k}\right), \quad i, j \in\left\{1, \ldots, \frac{1}{\varepsilon_{k}}\right\} .
$$



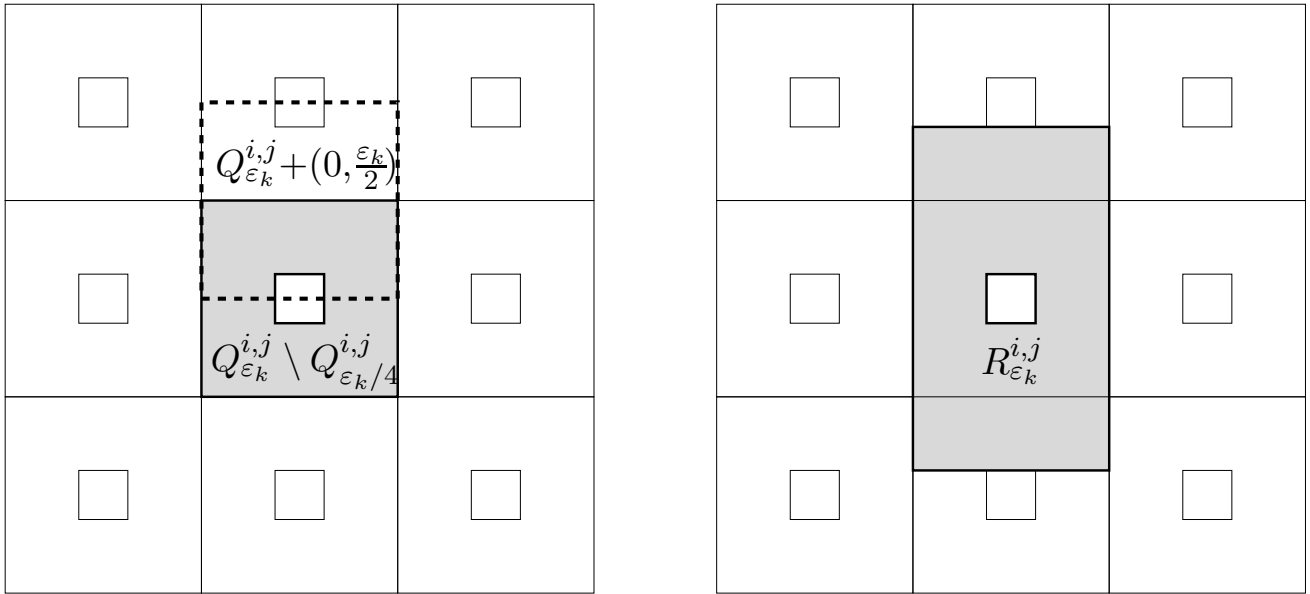

FiguRE 6 . In grey the sets $Q_{\varepsilon_{k}}^{i, j} \backslash Q_{\varepsilon_{k} / 4}^{i, j}$ and $R_{\varepsilon_{k}}^{i, j}$. The dashed line indicates the boundary of the translated square $Q_{\varepsilon_{k}}^{i, j}+\left(0, \varepsilon_{k} / 2\right)$.

We also consider the smaller squares $Q_{\varepsilon_{k} / 4}^{i, j}:=Q_{\varepsilon_{k} / 4}+p_{k}^{i, j}$ and the strips

$$
\begin{aligned}
& T_{\varepsilon_{k}}^{j}:=\left(\left(-\frac{1}{2}, \frac{1}{2}\right) \times\left(-\frac{\varepsilon_{k}}{2}, \frac{\varepsilon_{k}}{2}\right)\right)+\left(0,-\frac{1}{2}+\left(j-\frac{1}{2}\right) \varepsilon_{k}\right), \\
& T_{\frac{\varepsilon_{k}}{2}}^{j}:=\left(\left(-\frac{1}{2}, \frac{1}{2}\right) \times\left(-\frac{\varepsilon_{k}}{4}, \frac{\varepsilon_{k}}{4}\right)\right)+\left(0,-\frac{1}{2}+\left(j-\frac{1}{2}\right) \varepsilon_{k}\right) .
\end{aligned}
$$

Moreover, we define the sets $R_{\varepsilon_{k}}^{i, j}:=\varepsilon_{k} R+p_{k}^{i, j}$ (see Figure 6), where

$$
R:=((-1 / 2,1 / 2) \times(-7 / 8,7 / 8)) \backslash \bar{Q}_{1 / 4} .
$$

Let $\mathcal{J}_{k} \subset\left\{2, \ldots, \varepsilon_{k}^{-1}-1\right\}$ be the set of indices $j$ such that

$$
M S\left(u_{k}, R_{\varepsilon_{k}}^{1, j}\right) \leq \beta \varepsilon_{k},
$$

where $\beta>0$ will be chosen later. Thanks to the periodicity of $u_{k}$, for $j \in \mathcal{J}_{k}$ we have

$$
M S\left(u_{k}, R_{\varepsilon_{k}}^{i, j}\right) \leq \beta \varepsilon_{k},
$$

for every $i \in\left\{1, \ldots, \varepsilon_{k}^{-1}\right\}$.

For $\beta$ small enough, by scaling and translation (see also Remark 6) we can apply Lemma 5 in each square $Q_{\varepsilon_{k}}^{i, j} \pm\left(0, \varepsilon_{k} / 2\right)$, for $i \in\left\{1, \ldots, \varepsilon_{k}^{-1}\right\}$ and $j \in \mathcal{J}_{k}$, thus obtaining a new sequence $\left(v_{k}\right)$ which is equal to $\left(u_{k}\right)$ (at least) in $Q_{\varepsilon_{k} / 4}^{i, j}$ and is constant in the translated strips $T_{\varepsilon_{k} / 2}^{j}+\left(0, \varepsilon_{k} / 2\right)$, $T_{\varepsilon_{k} / 2}^{j}-\left(0, \varepsilon_{k} / 2\right)$ (the dark grey part in Figure $7(\mathrm{i})$ ). We call these two constant values $b_{k}^{j}$ and $a_{k}^{j}$, respectively. Moreover, Lemma 5 yields the existence of a constant $\alpha>0$ such that

$$
\alpha \int_{Q_{k}}\left|\nabla v_{k}\right|^{2} \mathrm{~d} x+\mathcal{H}^{1}\left(S_{v_{k}} \cap Q_{k}\right) \leq M S\left(u_{k}, Q_{k}\right),
$$

where $Q_{k}:=Q \cap \varepsilon_{k} P$ is the stiff matrix, cf. (2.7). Note that $v_{k}=u_{k}$ in $Q \backslash \varepsilon_{k} P$.

Due to the lack of control on $\nabla u_{k}$ in the soft inclusions $Q \backslash \varepsilon_{k} P$, it is not obvious that $v_{k} \rightarrow u_{t}$ in $L^{1}(Q)$. Then, to prove the desired convergence we employ a result from [5], originally stated for perforated domains. In fact, by [5, Theorem 1] one can find a sequence $\left(\bar{v}_{k}\right)$ such that $\bar{v}_{k}=v_{k}$ 
in $Q_{k}$ and $\bar{v}_{k} \rightarrow u_{t}$ strongly in $L^{1}(Q)$ (up to subsequences not relabelled). Hence,

$$
\int_{Q}\left|v_{k}-u_{t}\right| \mathrm{d} x=\int_{Q_{k}}\left|\bar{v}_{k}-u_{t}\right| \mathrm{d} x+\int_{Q \backslash \varepsilon_{k} P}\left|u_{k}-u_{t}\right| \mathrm{d} x \rightarrow 0,
$$

and the convergence to $u_{t}$ is preserved.

Step 2. In this step we modify $v_{k}$ in each strip $T_{\varepsilon_{k} / 2}^{j}$, for $j \in \mathcal{J}_{k}$, by replacing it with the affine interpolation between the values $a_{k}^{j}$ and $b_{k}^{j}$.

Denote by $v_{k}^{\text {aff }}$ the function defined in $\bigcup_{j \in \mathcal{J}_{k}} T_{\varepsilon_{k} / 2}^{j}$ (light grey part in Figure 8(i)) which is constant in its first variable and is the affine interpolation between $a_{k}^{j}$ and $b_{k}^{j}$ in its second variable. We set

$$
\tilde{v}_{k}:= \begin{cases}v_{k}^{\text {aff }} & \text { in } \bigcup_{j \in \mathcal{J}_{k}} T_{\frac{\varepsilon_{k}}{2}}^{j}, \\ v_{k} & \text { otherwise in } Q .\end{cases}
$$

For each $x_{1} \in(-1 / 2,1 / 2)$ let $S_{x_{1}}^{j}$ be the vertical one-dimensional slice of $T_{\varepsilon_{k} / 2}^{j}$ through $x_{1}$; i.e.,

$$
S_{x_{1}}^{j}:=\left(\left\{x_{1}\right\} \times\left[-\frac{\varepsilon_{k}}{4}, \frac{\varepsilon_{k}}{4}\right]\right)+\left(0,-\frac{1}{2}+\left(j-\frac{1}{2}\right) \varepsilon_{k}\right) .
$$

By [2, Theorems 3.28, 3.107, and 3.108], for $\mathcal{L}^{1}$-a.e. $x_{1}$ such that $S_{x_{1}}^{j} \cap S_{v_{k}}=\varnothing$ the function $v_{k}\left(x_{1}, \cdot\right)$ is absolutely continuous and therefore

$$
\left|b_{k}^{j}-a_{k}^{j}\right| \leq \int_{S_{x_{1}}^{j}}\left|\partial_{x_{2}} v_{k}\left(x_{1}, s\right)\right| \mathrm{d} s .
$$

Appealing to Jensen's inequality, we get

$$
\int_{T_{\frac{\varepsilon_{k}}{2}}^{j}}\left|\nabla v_{k}^{\mathrm{aff}}\right|^{2} \mathrm{~d} x=\frac{2}{\varepsilon_{k}}\left|b_{k}^{j}-a_{k}^{j}\right|^{2} \leq \int_{S_{x_{1}}^{j}}\left|\partial_{x_{2}} v_{k}\left(x_{1}, s\right)\right|^{2} \mathrm{~d} s
$$

for $x_{1}$ as above. Let

$$
\hat{P}:=\mathbb{R}^{2} \backslash\left(\left(\bigcup_{i \in \mathbb{Z}}\left(-\frac{1}{8}, \frac{1}{8}\right)+i\right) \times \mathbb{R}\right) ;
$$

we notice that the vertical one-dimensional slices of $\hat{P}$ do not intersect any of the soft inclusions. We now assume $\beta \leq 1 / 4$, so that at least half of the vertical one-dimensional slices in $T_{\varepsilon_{k} / 2}^{j} \cap \varepsilon_{k} \hat{P}$ (see Figure $7\left(\right.$ ii) ) do not intersect $S_{v_{k}}$, indeed

$$
\mathcal{H}^{1}\left(\left\{x_{1}: S_{x_{1}}^{j} \subset \varepsilon_{k} \hat{P} \text { and } S_{x_{1}}^{j} \cap S_{v_{k}}=\varnothing\right\}\right) \geq \frac{3}{4}-\beta \geq \frac{1}{2},
$$

where we have used the fact that for $j \in \mathcal{J}_{k}$

$$
\mathcal{H}^{1}\left(S_{v_{k}} \cap T_{\frac{\varepsilon_{k}}{2}}^{j} \cap \varepsilon_{k} \hat{P}\right) \leq \mathcal{H}^{1}\left(S_{u_{k}} \cap T_{\frac{\varepsilon_{k}}{2}}^{j} \cap \varepsilon_{k} \hat{P}\right) \leq \beta .
$$

Integrating (4.6) on $\left\{x_{1}: S_{x_{1}}^{j} \subset \varepsilon_{k} \hat{P}\right.$ and $\left.S_{x_{1}}^{j} \cap S_{v_{k}}=\varnothing\right\}$ we find

$$
\frac{1}{2} \int_{T_{\frac{\varepsilon_{k}}{2}}^{j}}\left|\nabla v_{k}^{\mathrm{aff}}\right|^{2} \mathrm{~d} x \leq \int_{T_{\frac{\varepsilon_{k}}{2}}^{j} \cap \varepsilon_{k} \hat{P}}\left|\nabla v_{k}\right|^{2} \mathrm{~d} x .
$$

Define

$$
C:=\bigcup\left\{\overline{T_{\varepsilon_{k}}^{j}}: j \in \mathcal{J}_{k}\right\}
$$

and consider the open set $A:=\operatorname{int}(C)$. By construction, we have

$$
\frac{1}{2} \int_{A}\left|\nabla \tilde{v}_{k}\right|^{2} \mathrm{~d} x \leq \int_{A \cap \varepsilon_{k} P}\left|\nabla v_{k}\right|^{2} \mathrm{~d} x .
$$




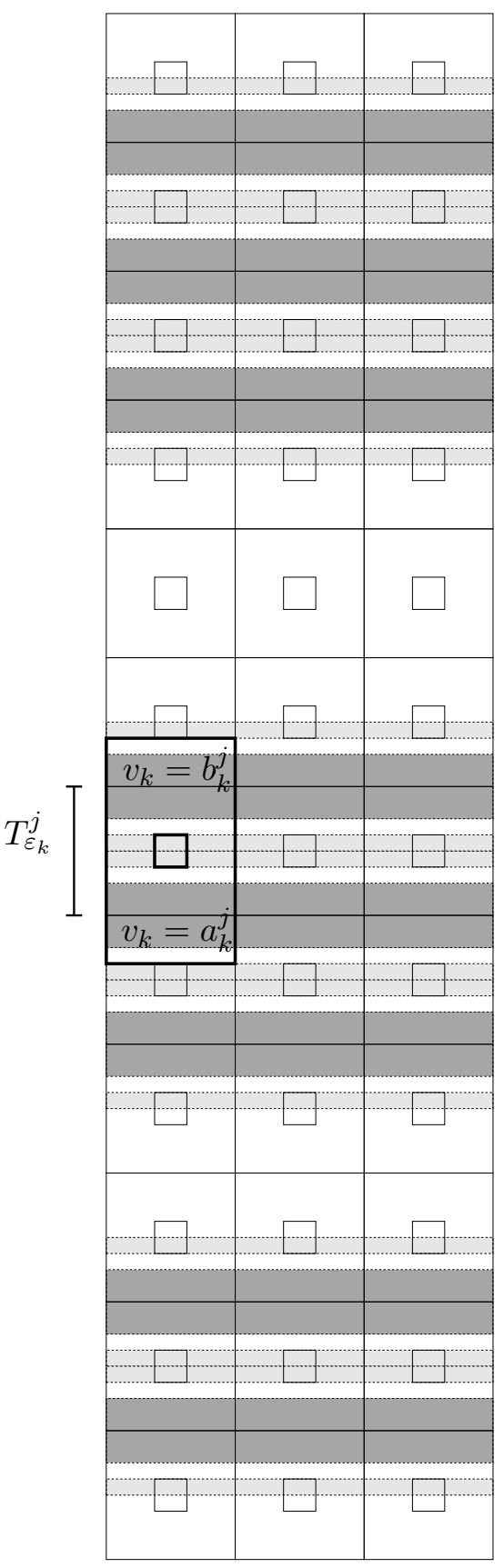

(i)

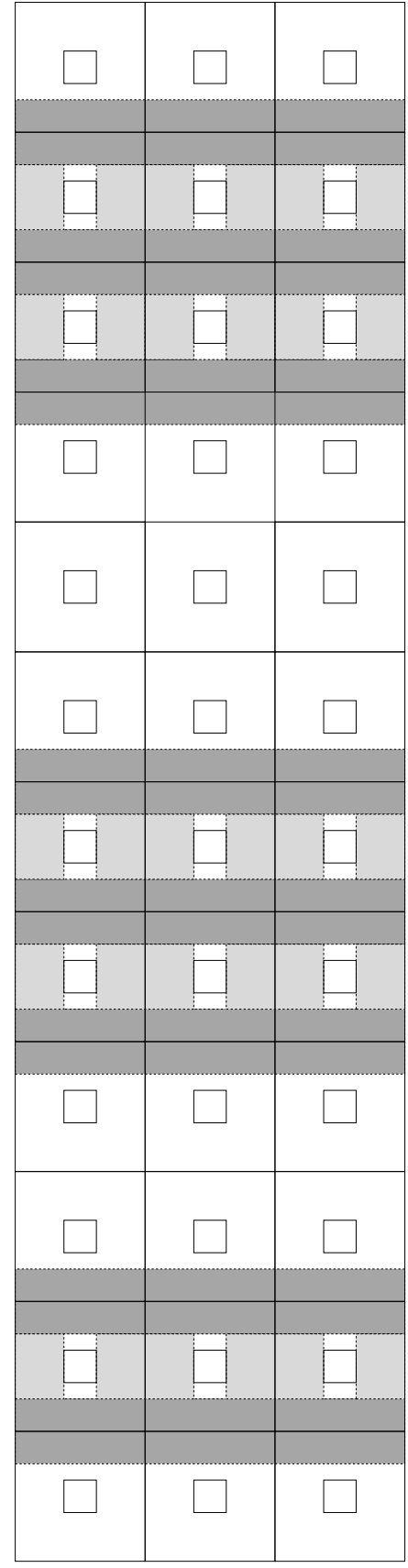

(ii)

Figure 7. (i) In black the boundary of the set $R_{\varepsilon_{k}}^{i, j}$. The function $v_{k}$ is defined in Step 1 and assumes constant values in each dark grey strip. (ii) In light grey the intersection of $\varepsilon_{k} \hat{P}$ with the strips $\bigcup_{j \in \mathcal{J}_{k}} T_{\varepsilon_{k} / 2}^{j}$. 


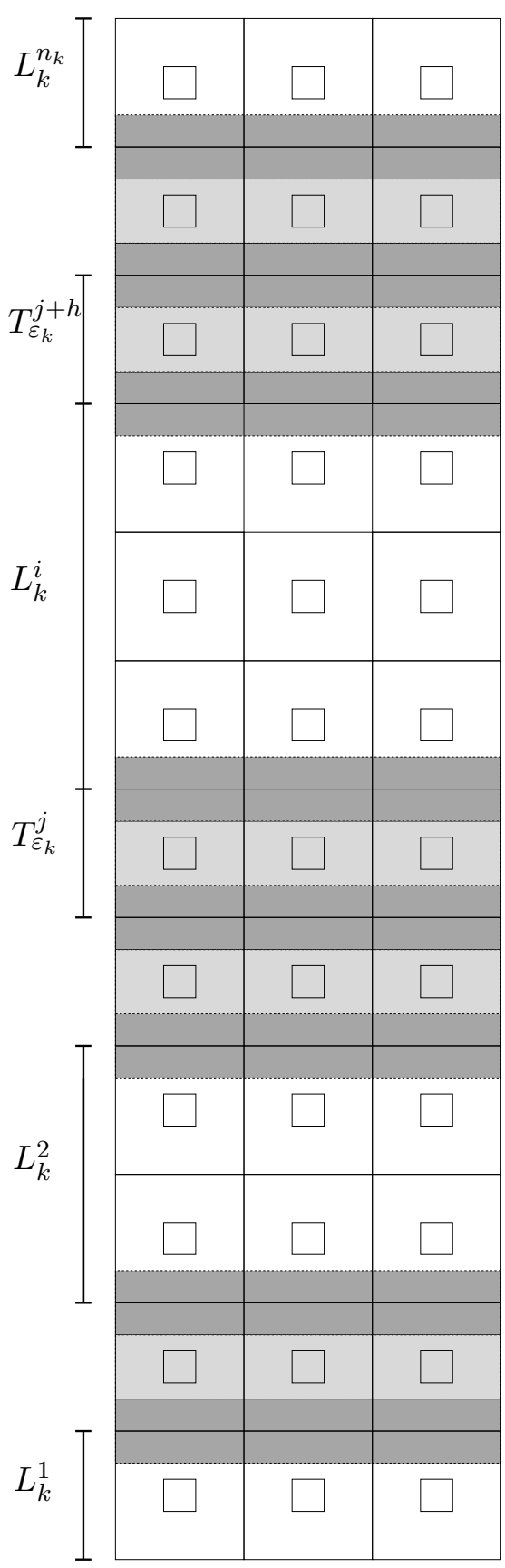

(i)

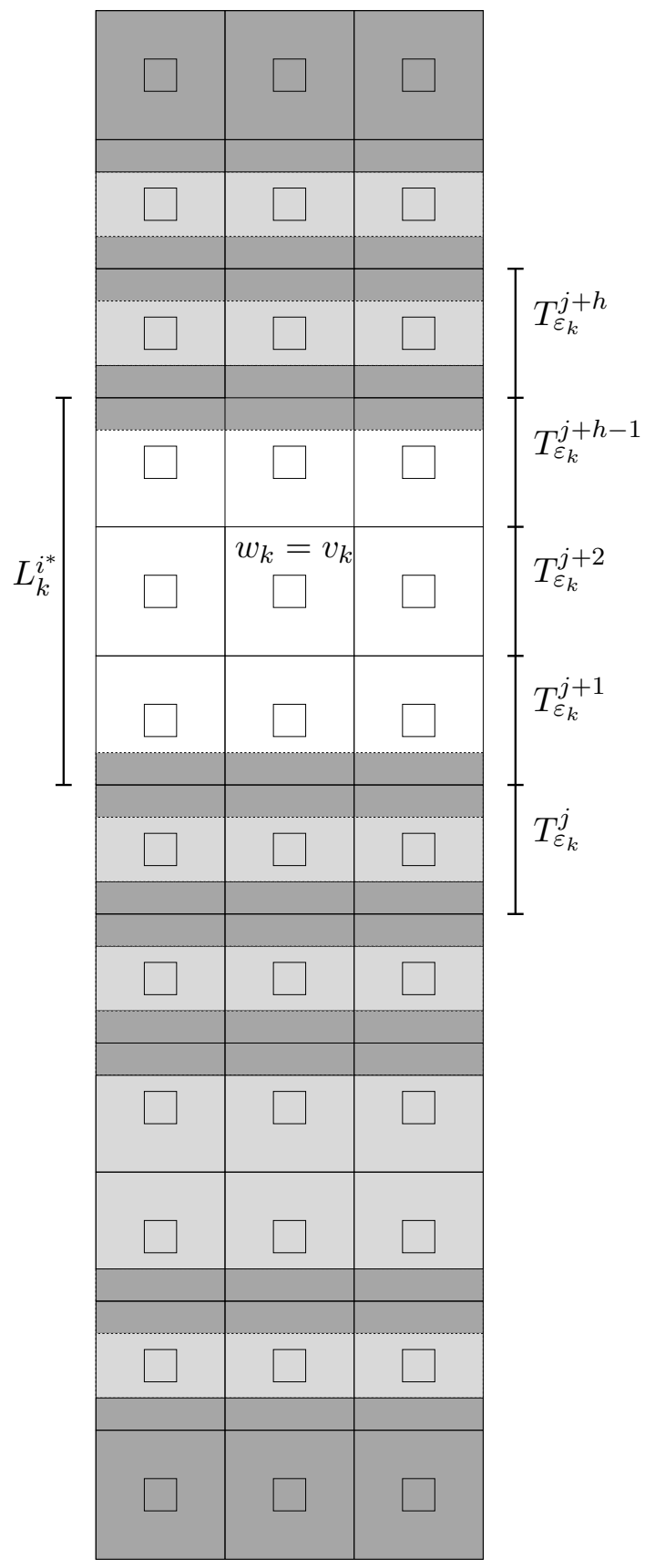

(ii)

Figure 8. (i) The function $\tilde{v}_{k}$ is defined in Step 2 and is affine in the light grey strips. (ii) The function $w_{k}$ is defined in Step 3; it assumes constant values in each dark grey strip and is affine in the light grey strips. Note that the surface energy concentrates in the strip $L_{\varepsilon_{k}}^{i^{*}}$. 
We want to show that $\tilde{v}_{k} \rightarrow u_{t}$ in $L^{1}(Q)$. To this end we introduce the auxiliary sequence $\left(\hat{v}_{k}\right)$ defined as

$$
\hat{v}_{k}:= \begin{cases}\tilde{v}_{k} & \text { in } A \\ 0 & \text { in } Q \backslash A .\end{cases}
$$

Denote by $L_{k}^{1}, \ldots, L_{k}^{n_{k}}$ the connected components of $Q \backslash A$ (see Figure 8(i)), enumerated going from the bottom to the top of $Q$. By definition of $\mathcal{J}_{k}$, we have that $T_{\varepsilon_{k}}^{1} \subset L_{k}^{1}$ and $T_{\varepsilon_{k}}^{\varepsilon_{k}^{-1}} \subset L_{k}^{n_{k}}$. Let $\mathcal{J}_{k}^{c}$ be the complement of $\mathcal{J}_{k}$ in $\left\{1, \ldots, \varepsilon_{k}^{-1}\right\}$. For $i \in\left\{1, \ldots, \varepsilon_{k}^{-1}\right\}$ and $j \in \mathcal{J}_{k}^{c}$ we have

$$
M S\left(u_{k}, R_{\varepsilon_{k}}^{i, j}\right)>\beta \varepsilon_{k} \text {. }
$$

Thus, recalling that $\liminf _{k} F_{\varepsilon_{k}}\left(u_{k}\right) \leq 1$, for $k$ large enough we deduce

$$
n_{k} \leq \# \mathcal{J}_{k}^{c} \leq \frac{2 F_{\varepsilon_{k}}\left(u_{k}\right)}{\beta} \leq \frac{3}{\beta}
$$

where $\# \mathcal{J}_{k}^{c}$ denotes the number of elements of $J_{k}^{c}$ and the prefactor 2 comes from the partial superposition of the sets $R_{\varepsilon_{k}}^{i, h}$. We clearly have $\left(\hat{v}_{k}\right) \subset S B V^{2}(Q)$ with discontinuities concentrated along the boundaries of $L_{j}^{h}$. Gathering (4.5), (4.7), and (4.8), we have that $M S\left(\hat{v}_{k}, Q\right)$ is bounded. Therefore, appealing to the compactness result [2, Theorem 4.8], we deduce that $\left(\hat{v}_{k}\right)$ is pre-compact in $L^{1}(Q)$. Since by definition of $\hat{v}_{k}$

$$
\tilde{v}_{k}= \begin{cases}\hat{v}_{k} & \text { in } A \\ v_{k} & \text { in } Q \backslash A,\end{cases}
$$

we have that also $\left(\tilde{v}_{k}\right)$ is pre-compact in $L^{1}(Q)$. Further, since $\tilde{v}_{k}=v_{k}$ on $T_{\varepsilon_{k} / 2}^{j} \pm\left(0, \varepsilon_{k} / 2\right)$, invoking Lemma 2 gives $\tilde{v}_{k} \rightarrow u_{t}$ in $L^{1}(Q)$.

Step 3. We now introduce the final sequence $\left(w_{k}\right)$ as a further modification of the sequence $\left(\tilde{v}_{k}\right)$; specifically, we set $w_{k}:=\tilde{v}_{k}$ in $A$ and define it in $Q \backslash A$ as explained below. We start observing that since

$$
\mathcal{L}^{2}(Q \backslash A)=\varepsilon_{k} \# \mathcal{J}_{k}^{c} \leq \frac{3 \varepsilon_{k}}{\beta} \rightarrow 0,
$$

this last modification will keep the limit $u_{t}$. Therefore we only need to check that we can also control the energy along the new sequence in terms of $F_{\varepsilon_{k}}\left(u_{k}, Q\right)$.

We start defining $w_{k}$ in $L_{k}^{1}$ and $L_{k}^{n_{k}}$. To this end let $m_{k}:=\min \mathcal{J}_{k}$ and $M_{k}:=\max \mathcal{J}_{k}$ and set $w_{k}:=a_{k}^{m_{k}}$ in $L_{k}^{1}$ and $w_{k}:=b_{k}^{M_{k}}$ in $L_{k}^{n_{k}}$. Since in this way we have not added any new discontinuities, this first modification of $\tilde{v}_{k}$ does not increase the energy.

Consider now an intermediate strip $L_{k}^{i}, i \in\left\{2, \ldots, n_{k}-1\right\}$. Observe that here $\tilde{v}_{k}=v_{k}$. By definition, $L_{k}^{i}$ is adjacent to two strips $T_{\varepsilon_{k}}^{j}$ and $T_{\varepsilon_{k}}^{j+h}$ for some $j, j+h \in \mathcal{J}_{k}$ (see Figure 8(i)). If $\mathcal{H}^{1}\left(S_{v_{k}} \cap L_{k}^{i}\right)<1 / 2$, we argue as in Step 2 and define $w_{k}$ in $L_{k}^{i}$ as the affine interpolation between the values $b_{k}^{j}$ and $a_{k}^{j+h}$, obtaining

$$
\frac{1}{4} \int_{L_{k}^{i}}\left|\nabla w_{k}\right|^{2} \mathrm{~d} x \leq \int_{L_{k}^{i} \cap \varepsilon_{k} \hat{P}}\left|\nabla v_{k}\right|^{2} \mathrm{~d} x .
$$

If $\mathcal{H}^{1}\left(S_{v_{k}} \cap L_{k}^{i}\right) \geq 1 / 2$, we simply set $w_{k}:=v_{k}$ in $L_{k}^{i}$. We notice that if there are two or more strips $L_{k}^{i}$ such that $\mathcal{H}^{1}\left(S_{v_{k}} \cap L_{k}^{i}\right) \geq 1 / 2$, then

$$
\int_{Q_{k}}\left|\nabla u_{k}\right|^{2} \mathrm{~d} x+\mathcal{H}^{1}\left(S_{u_{k}} \cap Q\right) \geq 1
$$


and the lower bound (4.3) holds true. On the other hand, there must be at least one of such strips; otherwise, $w_{k}$ would be piecewise affine (thus Sobolev) in the whole $Q$, so in view of (4.5), (4.7), and (4.9) we should get

$$
\frac{\alpha}{4} \int_{Q}\left|\nabla w_{k}\right|^{2} \mathrm{~d} x \leq F_{\varepsilon_{k}}\left(u_{k}, Q\right),
$$

and then deduce that $\left(w_{k}\right)$ converges weakly in $H^{1}(Q)$, thus violating the $L^{1}(Q)$-convergence to $u_{t}$. Therefore, we can assume that there is precisely one index $i^{*}$ for which $\mathcal{H}^{1}\left(S_{v_{k}} \cap L_{k}^{i^{*}}\right) \geq 1 / 2$.

Summarising, in this step we have defined a sequence $\left(w_{k}\right)$ that is piecewise affine in $Q \backslash L_{k}^{i^{*}}$ (see Figure 8(ii)). In particular $w_{k}=b_{k}^{j}$ in $T_{\varepsilon_{k} / 2}^{j}+\left(0, \varepsilon_{k} / 2\right)$ and $w_{k}=a_{k}^{j+h}$ in $T_{\varepsilon_{k} / 2}^{j+h}-\left(0, \varepsilon_{k} / 2\right)$. Moreover, by construction $w_{k} \rightarrow u_{t}$ strongly in $L^{1}(Q)$.

Step 4. In this step we show that the energy of $w_{k}$ concentrates in the strip $L_{k}^{i^{*}}$ and provides us with a lower bound on $F_{\varepsilon_{k}}\left(u_{k}, Q\right)$ as in (4.3).

To this end, we start observing that since $\hat{P} \subset P$ and $w_{k}=v_{k}$ in $L_{k}^{i^{*}}$, for $k$ large enough (so that $\left.\varepsilon_{k}<\alpha\right)$, by $(4.5)$ we have

$$
F_{\varepsilon_{k}}\left(u_{k}, Q\right) \geq \alpha \int_{L_{k}^{i^{*}} \cap \varepsilon_{k} \hat{P}}\left|\nabla w_{k}\right|^{2} \mathrm{~d} x+\varepsilon_{k} \int_{L_{k}^{i^{*}} \backslash \varepsilon_{k} \hat{P}}\left|\nabla w_{k}\right|^{2} \mathrm{~d} x+\mathcal{H}^{1}\left(S_{w_{k}} \cap L_{k}^{i^{*}}\right) .
$$

Since $j+1, \ldots, j+h-1 \in \mathcal{J}_{k}^{c}$ (see Figure 8(ii)), similarly to (4.8), we get

$$
h-1 \leq \frac{2 \mathcal{H}^{1}\left(S_{w_{k}} \cap Q\right)}{\beta} \leq \frac{3}{\beta} .
$$

Moreover, since $w_{k} \rightarrow u_{t}$ strongly in $L^{1}(Q)$ and outside the strip $L_{k}^{i^{*}}$ the function $w_{k}$ is piecewise affine with gradient uniformly bounded in $L^{2}\left(Q ; \mathbb{R}^{2}\right)$, necessarily $L_{k}^{i^{*}}$ converges to $\left\{x_{2}=0\right\} \cap Q$ in the sense of the Hausdorff distance and $b_{k}^{j}-a_{k}^{j+h} \rightarrow t$ as $k \rightarrow+\infty$; hence in particular

$$
\left|b_{k}^{j}-a_{k}^{j+h}\right| \geq \frac{t}{2}
$$

for $k$ large enough. Comparing the bulk energy of $w_{k}$ in $L_{k}^{i^{*}} \cap \varepsilon_{k} \hat{P}$ with that of the affine interpolation between the values $b_{k}^{j}$ and $a_{k}^{j+h}$ we get

$$
\int_{L_{k}^{i^{*}} \cap \varepsilon_{k} \hat{P}}\left|\nabla w_{k}\right|^{2} \mathrm{~d} x \geq\left(\frac{3}{4}-\mathcal{H}^{1}\left(S_{w_{k}} \cap L_{k}^{i^{*}} \cap \varepsilon_{k} \hat{P}\right)\right) \frac{\left|b_{k}^{j}-a_{k}^{j+h}\right|^{2}}{\varepsilon_{k}(h-1)} .
$$

Since

$$
\varepsilon_{k}(h-1)=\mathcal{L}^{2}\left(L_{k}^{i^{*}}\right) \leq \mathcal{L}^{2}(Q \backslash A) \rightarrow 0,
$$

gathering (4.4), (4.11), and (4.12) we have

$$
\liminf _{k \rightarrow+\infty} \mathcal{H}^{1}\left(S_{w_{k}} \cap L_{k}^{i^{*}} \cap \varepsilon_{k} \hat{P}\right) \geq \frac{3}{4}
$$

We now estimate the energy in $L_{k}^{i^{*}} \backslash \varepsilon_{k} \hat{P}$. When we come to minimise

$$
\hat{F}\left(w_{k}\right):=\varepsilon_{k} \int_{L_{k}^{i^{*}} \backslash \varepsilon_{k} \hat{P}}\left|\nabla w_{k}\right|^{2} \mathrm{~d} x+\mathcal{H}^{1}\left(S_{w_{k}} \cap\left(L_{k}^{i^{*}} \backslash \varepsilon_{k} \hat{P}\right)\right),
$$

by an easy one-dimensional argument we find that in each connected component of $L_{k}^{i^{*}} \backslash \varepsilon_{k} \hat{P}$ the minimum is attained either at the affine interpolation between the two constants, or at a 


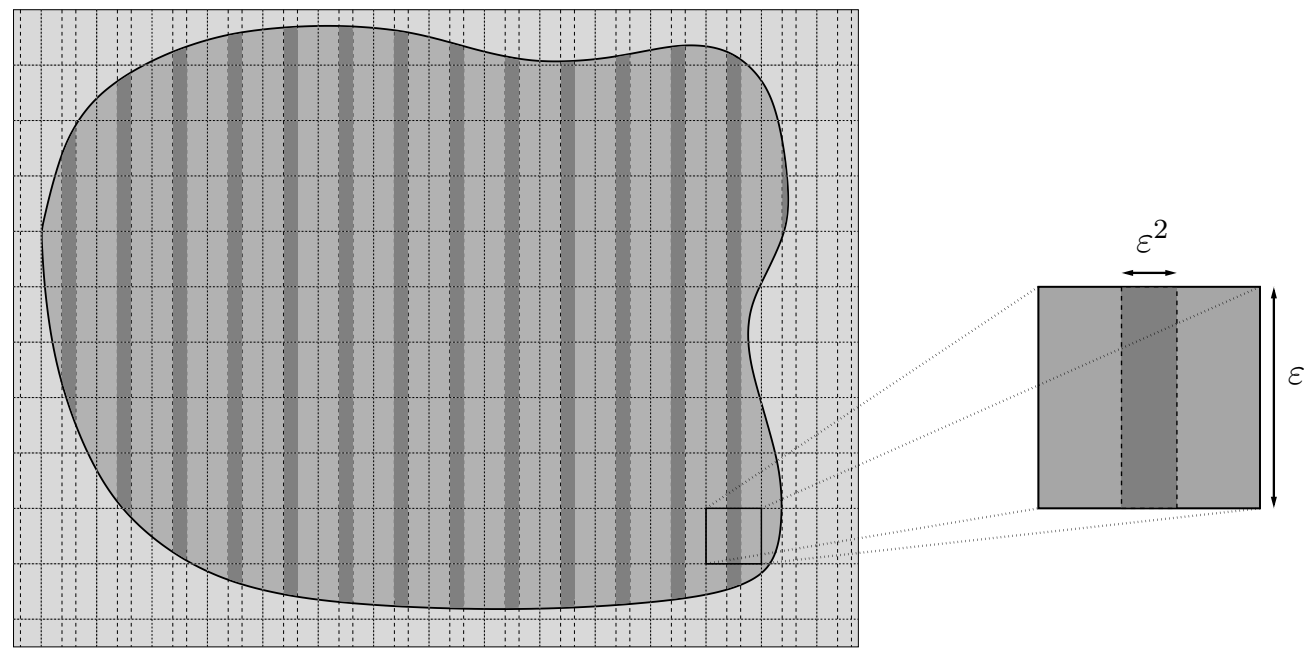

FiguRE 9. In darker grey the reinforcement $\Omega \backslash \varepsilon D_{\varepsilon}$.

function that is piecewise constant, takes values in $\left\{b_{k}^{j}, a_{k}^{j+h}\right\}$, and jumps along a horizontal line. Computing the energy of these two competitors leads to the estimate

$$
\hat{F}\left(w_{k}\right) \geq \min \left\{\frac{\left|b_{k}^{j}-a_{k}^{j+h}\right|^{2}}{4(h-1)}, \frac{1}{4}\right\} .
$$

Hence, finally, combining (4.10)-(4.14) gives

$$
\liminf _{k} F_{\varepsilon_{k}}\left(u_{k}\right) \geq \frac{3}{4}+\min \left\{\frac{\beta}{48} t^{2}, \frac{1}{4}\right\}=\min \left\{\frac{3}{4}+\frac{\beta}{48} t^{2}, 1\right\}
$$

and thus (4.3) with $c_{3}=\beta / 48$.

We now remove the assumption that $\varepsilon_{k}^{-1}$ is an odd integer. To this end, let $m_{k}$ be the largest integer such that $\left(2 m_{k}+1\right) \varepsilon_{k} \leq 1$. Since $Q_{\left(2 m_{k}+1\right) \varepsilon_{k}}$ can be decomposed into periodicity cells entirely contained in $Q$, we can repeat in $Q_{\left(2 m_{k}+1\right) \varepsilon_{k}}$ the construction leading to the sequence $\left(w_{k}\right)$. Finally, we extend $w_{k}$ by reflection in the vanishing frame $Q \backslash Q_{\left(2 m_{k}+1\right) \varepsilon_{k}}$ and conclude.

\section{Homogenisation of A fibre-Reinforced BRittle Composite}

The object of this last section is the homogenisation of a laminar composite made of a brittle constituent reinforced by periodically distributed tougher fibres (see Figure 9). Although from a modelling point of view this problem is quite different from the one analysed in the previous sections, we show that the same mathematical techniques apply also in this case (with minor modifications) and lead again to a homogenised model of cohesive type.

Let $\Omega$ be a bounded open subset of $\mathbb{R}^{2}$ with Lipschitz boundary. For any $\varepsilon>0$ we consider the energy-functional $E_{\varepsilon}: L^{1}(\Omega) \rightarrow[0,+\infty]$ defined as

$$
E_{\varepsilon}(u):= \begin{cases}\int_{\Omega}|\nabla u|^{2} \mathrm{~d} x+\mathcal{H}^{1}\left(S_{u} \cap \Omega \cap \varepsilon D_{\varepsilon}\right)+\frac{1}{\varepsilon} \mathcal{H}^{1}\left(S_{u} \cap\left(\Omega \backslash \varepsilon D_{\varepsilon}\right)\right) & \text { if } u \in S B V^{2}(\Omega), \\ +\infty & \text { otherwise in } L^{1}(\Omega),\end{cases}
$$

where

$$
D_{\varepsilon}:=\mathbb{R}^{2} \backslash \bigcup_{i \in \mathbb{Z}}\left(A_{\varepsilon}+(i, 0)\right), \quad A_{\varepsilon}:=\left(-\frac{\varepsilon}{2}, \frac{\varepsilon}{2}\right) \times \mathbb{R}
$$


Here, $\Omega \backslash \varepsilon D_{\varepsilon}$ (constituted by vertical strips of thickness $\varepsilon^{2}$ ) models the so-called fibre-reinforcement. Therefore, unlike the previous case, the two constituents $\Omega \cap \varepsilon D_{\varepsilon}$ and $\Omega \backslash \varepsilon D_{\varepsilon}$ now differ in their toughness, while they share (for simplicity) the same elastic modulus, equal to one; however, the same qualitative behaviour described below is retrieved when the two constituents have different elastic moduli (both independent of $\varepsilon$ ).

We notice that following $[4,21]$ one could also model the tough fibres as a constituent with infinite toughness (thus imposing a restriction on the location of the jump set of the admissible displacements). We rather find less artificial to model the reinforcement as a material whose toughness diverges as $\varepsilon$ tends to zero. Further, in order to obtain a nontrivial limit model we require that the toughness is equal to the inverse of the length-scale of the microstructure. Indeed, different choices lead to a $\Gamma$-limit where the surface energy density either is identically equal to one or grows linearly with the crack-opening. (This can be seen for instance arguing as in the estimate from above in the proof of Theorem 5.) When the toughness equals $1 / \varepsilon$ we prove instead that the surface energy density depends on the crack-opening and becomes constant when the crack-opening is large (see (5.4)).

The following result holds true.

Theorem 5. For every decreasing sequence of positive numbers converging to zero, there exists a subsequence $\left(\varepsilon_{k}\right)$ such that $\left(E_{\varepsilon_{k}}\right) \Gamma$-converges to a functional $E: L^{1}(\Omega) \rightarrow[0,+\infty]$ of the form

$$
E(u):= \begin{cases}\int_{\Omega}|\nabla u|^{2} \mathrm{~d} x+\int_{S_{u}} g\left([u], \nu_{u}\right) \mathrm{d} \mathcal{H}^{1} & \text { if } u \in G S B V^{2}(\Omega), \\ +\infty & \text { otherwise in } L^{1}(\Omega),\end{cases}
$$

where $g: \mathbb{R} \times \mathbb{S}^{1} \rightarrow[0,+\infty)$ is a Borel function satisfying the following properties:

(i) for any fixed $\nu \in \mathbb{S}^{1}, g(\cdot, \nu)$ is nondecreasing in $(0,+\infty)$ and satisfies the symmetry condition $g(-t,-\nu)=g(t, \nu) ;$ moreover, for every $t \neq 0$ and $\nu \in \mathbb{S}^{1}$

$$
1 \leq g(t, \nu) \leq 2
$$

(ii) for every $t>0$

$$
\min \left\{1+c t^{2}, 2\right\} \leq g\left(t, e_{2}\right) \leq \min \{1+2 \sqrt{2} t, 2\},
$$

for some $c>0$. Hence in particular $g\left(0^{+}, e_{2}\right)=1$ and $g\left(t, e_{2}\right)=2$ for $t$ larger than some $t_{0}>0$.

We remark that (5.1) shares some similarities with the energy-functionals considered in [4, 21 where the authors analyse the asymptotic behaviour of a brittle material reinforced by a reticulated (connected) unbreakable structure, modelled as a constituent with infinite toughness. In $[4$, Theorem 3.1] and [21, Theorem 2.2] it is shown that a composite as above gives rise to a homogenised model of cohesive type (with an activation threshold). Moreover, the effect of having (suitably chosen) regions with infinite toughness is that the limit surface energy density grows linearly in the crack-opening, which is though unsatisfactory from a physical point of view. In this perspective, Theorem 5 provides us with a homogenised model which is physically more relevant than the one obtained in $[4,21]$.

The proof of Theorem 5 closely follows those of Theorems 1 and 2, for this reason in the present section we give just a sketch of this proof detailing only the parts which are substantially different.

$\Gamma$-convergence and integral representation. The $\Gamma$-convergence of the functionals $E_{\varepsilon}$ can be derived also in this case by combining the localisation method and the integral representation result [7, Theorem 1]. The main difference with the previous case is that now we trivially have $M S \leq E_{\varepsilon}$ while $E_{\varepsilon}$ does not satisfy an estimate from above in terms of the Mumford-Shah 
functional. Hence, to fully fit the assumptions needed to apply the localisation method of $\Gamma$ convergence, we set $E^{\prime \prime}:=\Gamma-\lim \sup _{\varepsilon \rightarrow 0} E_{\varepsilon}$ and prove that $E^{\prime \prime} \leq 2 M S$. To this end, it is convenient to introduce the following notation.

Let us fix an open rectangle $R$ containing $\bar{\Omega}$ and let $\mathcal{W}(R)$ be the space of all functions $w \in S B V^{2}(R) \cap L^{\infty}(R)$ enjoying the following properties:

- $S_{w} \subset L$, with $L$ finite union of pairwise disjoint closed segments contained in $R$;

- $w \in W^{1, \infty}(R \backslash L)$.

Moreover, we denote by $\mathcal{A}_{L}(\Omega)$ the class of all open subsets of $\Omega$ with Lipschitz boundary.

To obtain the desired estimate on $E^{\prime \prime}$ we need the following approximation lemma which is a consequence of [16, Theorem 3.9].

Lemma 6. Let $U \in \mathcal{A}_{L}(\Omega)$ and let $u \in S B V^{2}(U) \cap L^{\infty}(U)$. Then $u$ has an extension $v \in$ $S B V^{2}(R) \cap L^{\infty}(R)$ with compact support in $R$, such that

$$
\mathcal{H}^{1}\left(S_{v} \cap \partial U\right)=0
$$

and $\|v\|_{L^{\infty}(R)}=\|u\|_{L^{\infty}(U)}$. Moreover, there exist a sequence $\left(w_{j}\right) \subset \mathcal{W}(R)$ converging to $v$ in $L^{1}(R)$, and a sequence $\left(L_{j}\right)$ of finite unions of pairwise disjoint closed segments contained in $R$ and such that $S_{w_{j}} \subset L_{j}$, with the following properties:

$$
\begin{gathered}
\left\|w_{j}\right\|_{L^{\infty}(R)} \leq\|v\|_{L^{\infty}(R)}=\|u\|_{L^{\infty}(U)}, \\
\nabla w_{j} \rightarrow \nabla v \quad \text { strongly in } L^{2}\left(R ; \mathbb{R}^{2}\right), \text { hence } \nabla w_{j} \rightarrow \nabla u \quad \text { strongly in } L^{2}\left(U ; \mathbb{R}^{2}\right), \\
\mathcal{H}^{1}\left(L_{j} \cap U\right) \rightarrow \mathcal{H}^{1}\left(S_{v} \cap U\right)=\mathcal{H}^{1}\left(S_{u} \cap U\right) .
\end{gathered}
$$

Proof. To prove the first assertion we can use locally a reflection argument in a curvilinear coordinate system for which the boundary is flat. The global extension can be obtained, as usual, through a partition of unity. Then, the existence of the approximating sequence $\left(w_{j}\right)$ is a consequence of the density result [16, Theorem 3.9].

We are in a position to prove the following proposition.

Proposition 1. For every $u \in S B V^{2}(\Omega) \cap L^{\infty}(\Omega)$ and for every $U \in \mathcal{A}_{L}(\Omega)$ we have

$$
E^{\prime \prime}(u, U) \leq \int_{U}|\nabla u|^{2} \mathrm{~d} x+2 \mathcal{H}^{1}\left(S_{u} \cap U\right) .
$$

Proof. We fix $U \in \mathcal{A}_{L}(\Omega)$; in view of Lemma 6 and of the locality of $E^{\prime \prime}$ it is enough to prove

$$
E^{\prime \prime}(u, U) \leq \int_{U}|\nabla u|^{2} \mathrm{~d} x+2 \mathcal{H}^{1}(L \cap U)
$$

for $u \in \mathcal{W}(R)$.

We want to construct a sequence $\left(u_{k}\right) \subset S B V^{2}(U)$ converging to $u$ in $L^{1}(\Omega)$ and such that

$$
\limsup _{k \rightarrow+\infty} E_{\varepsilon_{k}}\left(u_{k}, U\right) \leq \int_{U}|\nabla u|^{2} \mathrm{~d} x+2 \mathcal{H}^{1}(L \cap U) .
$$

Since $U$ has Lipschitz boundary, we can slightly modify $u$ near each connected component of $\bar{S}_{u}$ to find a $L$ that intersects $\partial U$ in a finite number of points. This can be done, for instance, by slightly shifting these connected components taking into account the area formula for $\partial U$.

Now we explicitly construct $\left(u_{k}\right)$ when $L$ is a single closed segment; then, the general case follows easily. Let $\nu \in \mathbb{S}^{1}$ denote the normal to $L$. We need to distinguish two cases.

Case 1: $\nu=e_{1}$; i.e., $L$ is a vertical segment. 
To fix the ideas let $c \in \mathbb{R}$ and suppose that $L \subset\left\{x_{1}=c\right\}$. Let $R^{\prime}$ be an open rectangle such that $\bar{\Omega} \cup L \subseteq R^{\prime} \subset \subset R$. In this case we obtain $u_{k}$ as a suitable translation of $u$. The latter is needed in order to prevent the possibility that $L$ entirely falls in a tough vertical fibre, thus paying too much in energy. To this end, for $k$ large enough and for $x \in R^{\prime}$ we set

$$
u_{k}(x):=u\left(x+\left(c-\left\lfloor\frac{c}{\varepsilon_{k}}\right\rfloor \varepsilon_{k}-\varepsilon_{k}^{2}\right) e_{1}\right),
$$

so that $S_{u_{k}}$ is contained in the vertical line $x_{1}=\left\lfloor\frac{c}{\varepsilon_{k}}\right\rfloor \varepsilon_{k}+\varepsilon_{k}^{2}$. Hence, $\left(u_{k}\right) \subset S B V^{2}\left(R^{\prime}\right)$, $S_{u_{k}} \cap \Omega \subseteq \Omega \cap \varepsilon_{k} D_{\varepsilon_{k}}, u_{k}$ is bounded in $L^{\infty}\left(R^{\prime}\right), u_{k} \rightarrow u$ in $L^{1}(\Omega)$. Moreover,

$$
\begin{aligned}
\lim _{k \rightarrow+\infty} E_{k}\left(u_{k}, U\right) & =\lim _{k \rightarrow+\infty} \int_{U}\left|\nabla u_{k}\right|^{2} \mathrm{~d} x+\mathcal{H}^{1}\left(S_{u_{k}} \cap U \cap \varepsilon_{k} D_{\varepsilon_{k}}\right) \\
& \leq \int_{U}|\nabla u|^{2} \mathrm{~d} x+\mathcal{H}^{1}(L \cap U),
\end{aligned}
$$

which gives the desired estimate in this case.

Case 2: $\nu \neq e_{1}$.

Let $\vartheta$ be the angle (measured anticlockwise) between $e_{1}$ and $\nu^{\perp}$; thanks to the symmetries of the problem, it is enough to consider the case $0 \leq \vartheta<\pi / 2$.

Let $N_{k} \in \mathbb{N}$ be the number of tough fibres intersecting $L \cap U$; notice that since $L$ intersects $\partial U$ in a finite number of points, we have

$$
\frac{\varepsilon_{k}}{\cos \vartheta} N_{k} \leq \mathcal{H}^{1}(L \cap U)+o(1) \quad \text { as } k \rightarrow+\infty .
$$

Then, setting $u_{k}:=u$ for every $k \in \mathbb{N}$, in view of (5.6) we find

$$
\begin{aligned}
& \lim _{k \rightarrow+\infty} E_{k}\left(u_{k}, U\right) \\
& =\lim _{k \rightarrow+\infty}\left(\int_{U}\left|\nabla u_{k}\right|^{2} \mathrm{~d} x+\mathcal{H}^{1}\left(S_{u_{k}} \cap U \cap \varepsilon_{k} D_{\varepsilon_{k}}\right)+\frac{1}{\varepsilon_{k}} \mathcal{H}^{1}\left(S_{u_{k}} \cap\left(U \backslash \varepsilon_{k} D_{\varepsilon_{k}}\right)\right)\right) \\
& =\int_{U}|\nabla u|^{2} \mathrm{~d} x+\lim _{k \rightarrow+\infty}\left(N_{k} \frac{\varepsilon_{k}}{\cos \vartheta}+\frac{1}{\varepsilon_{k}} N_{k} \frac{\varepsilon_{k}^{2}}{\cos \vartheta}\right) \\
& \leq \int_{U}|\nabla u|^{2} \mathrm{~d} x+2 \mathcal{H}^{1}(L \cap U),
\end{aligned}
$$

and thus the thesis.

Thus by virtue of Proposition 1, arguing as in Section 3 we can deduce that $E_{\varepsilon} \Gamma$-converges to the functional $E$ as in (5.2). We also remark that the bound from above in (5.3) follows from (5.5) choosing

$$
u(x)=\left\{\begin{array}{ll}
t & \text { if } x \cdot \nu>0 \\
0 & \text { if } x \cdot \nu \leq 0
\end{array} \text { and } \quad U=Q\right.
$$

Dependence on the crack-opening. We now turn to the proof of (ii) Theorem 5 . We start proving the estimate from above in (5.4).

Estimate from above. We want to show that $g\left(t, e_{2}\right) \leq 1+2 \sqrt{2} t$. To this end, let $R_{\varepsilon} \subset A_{\varepsilon}$ be the open rectangle defined as

$$
R_{\varepsilon}:=\left(-\frac{\varepsilon}{2}, \frac{\varepsilon}{2}\right) \times\left(-\frac{t}{2 \sqrt{2}}, \frac{t}{2 \sqrt{2}}\right) .
$$




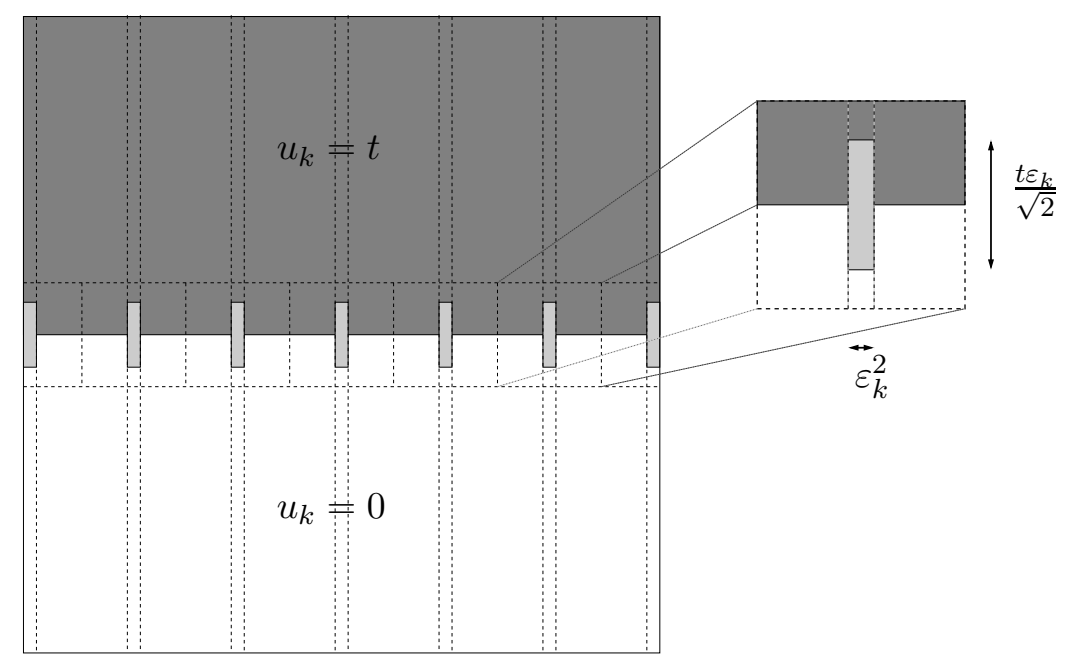

Figure 10. The optimal sequence $u_{k}$.

Set

$$
R_{k}:=Q \cap \varepsilon_{k}\left(\bigcup_{i \in \mathbb{Z}} R_{\varepsilon_{k}}+(i, 0)\right)
$$

and let $\left(u_{k}\right) \subset S B V^{2}(Q)$ be the sequence of functions defined as

$$
u_{k}(x):= \begin{cases}t & \text { if } x \in Q \backslash R_{k} \text { and } x_{2} \geq 0, \\ \frac{t}{2}+\frac{\sqrt{2}}{\varepsilon_{k}} x_{2} & \text { if } x \in R_{k}, \\ 0 & \text { if } x \in Q \backslash R_{k} \text { and } x_{2}<0,\end{cases}
$$

(see Figure 10). We clearly have $u_{k} \rightarrow u_{t}$ in $L^{1}(Q)$; moreover

$$
\int_{R_{k}}\left|\nabla u_{k}\right|^{2} \mathrm{~d} x \leq \varepsilon_{k}\left(\left\lfloor\frac{1}{\varepsilon_{k}}\right\rfloor+1\right)(\sqrt{2} t) \quad \text { and } \quad \mathcal{H}^{1}\left(S_{u_{k}}\right) \leq \varepsilon_{k}\left(\left\lfloor\frac{1}{\varepsilon_{k}}\right\rfloor+1\right)(1+\sqrt{2} t) .
$$

Since $S_{u_{k}} \subset Q \cap \varepsilon_{k} D_{\varepsilon_{k}}$, we readily deduce

$$
g\left(t, e_{2}\right)=E\left(u_{t}, Q\right) \leq \limsup _{k \rightarrow+\infty} E_{\varepsilon_{k}}\left(u_{k}, Q\right) \leq 1+2 \sqrt{2} t
$$

and hence the estimate from above.

Estimate from below. We now discuss the more delicate estimate from below in (5.4). Here we follow the steps of the proof of Theorem 2, from which we borrow some notation.

We notice that in this case the consecutive modifications of a sequence with equibounded energy are in general easier with respect to those in Theorem 2 . For instance, since $E_{\varepsilon}$ is bounded from below by the Mumford-Shah functional now we can readily deduce that the (analogues of the) two sequences $\left(v_{k}\right)$ and $\left(\tilde{v}_{k}\right)$ are precompact in $S B V^{2}(Q)$. On the other hand, in the present case, when we come to define (the analogous of) $v_{k}$ we cannot directly apply Lemma 5 . Indeed, to keep the control on the energy of $v_{k}$, now we need to avoid the introduction of new jumps falling in the reinforced fibres. For this reason we need a suitably improved variant of Lemma 5.

The following lemma is a slight modification of the patching lemma [4, Lemma 3.3], to which we refer for an idea of the proof, and it holds true in dimension two only. 
Lemma 7. There exist two constants $\alpha, \beta>0$ with the following property: Let $u \in S B V^{2}(Q) \cap$ $L^{\infty}(Q)$ be symmetric in the first variable and such that $\mathcal{H}^{1}\left(S_{u} \cap U\right) \leq \beta$, where $U:=(-1 / 2,1 / 2) \times$ $(-3 / 8,3 / 8)$. Then there exists a function $v \in S B V^{2}(Q) \cap L^{\infty}(Q)$ still symmetric and such that

(i) $v$ is constant in $(-1 / 2,1 / 2) \times(-1 / 4,1 / 4)$;

(ii) $v=u$ in $Q \backslash U$;

(iii) $\|v\|_{L^{\infty}(Q)} \leq\|u\|_{L^{\infty}(Q)}$;

(iv) $\alpha \int_{Q}|\nabla v|^{2} \mathrm{~d} x \leq \int_{Q}|\nabla u|^{2} \mathrm{~d} x$;

(v) $S_{v} \subset S_{u}$.

On account of Lemma 7 we are now ready to show that for any given $\left(u_{k}\right) \subset S B V^{2}(Q)$ such that $u_{k} \rightarrow u_{t}$ in $L^{1}(Q)$, we have

$$
\liminf _{k} E_{\varepsilon_{k}}\left(u_{k}\right) \geq \min \left\{1+c t^{2}, 2\right\}
$$

for some $c>0$.

We may assume that $\lim \inf _{k} E_{\varepsilon_{k}}\left(u_{k}\right) \leq 2$, otherwise there is nothing to prove. As in Theorem 2 , we can additionally assume that the liminf is actually a limit, that $0 \leq u_{k} \leq t$ in $Q$, that $\varepsilon_{k}^{-1}$ is an odd integer, and, in view of Lemma 3 , that $u_{k}$ is $\varepsilon_{k}$-periodic and symmetric in its first variable. Also the general strategy of the proof remains the same: we are going to modify $\left(u_{k}\right)$ obtaining two intermediate sequences $\left(v_{k}\right)$ and $\left(\tilde{v}_{k}\right)$, and then finally the more regular sequence $\left(w_{k}\right)$ whose energy concentrates in a horizontal layer of thickness proportional to $\varepsilon_{k}$.

Let $R_{\varepsilon_{k}}^{i, j}:=\varepsilon_{k} R+p_{k}^{i, j}$, where $R:=(-1 / 2,1 / 2) \times(-7 / 8,7 / 8)$, and let $\mathcal{J}_{k} \subset\left\{2, \ldots, \varepsilon_{k}^{-1}-1\right\}$ be the set of indices $j$ such that

$$
\mathcal{H}^{1}\left(S_{u_{k}} \cap R_{\varepsilon_{k}}^{1, j}\right) \leq \beta
$$

Therefore, proceeding as in Theorem 2 Step 1, we may now appeal to Lemma 7 to define a new sequence $\left(v_{k}\right)$ without introducing any new jumps, and thus keeping the control on the energy. Moreover, since $E_{\varepsilon} \geq M S$ the sequence $\left(v_{k}\right)$ is precompact in $S B V^{2}(Q)$.

Then, arguing as in Theorem 2 Step 2 , we further modify $\left(v_{k}\right)$ constructing a new sequence $\left(\tilde{v}_{k}\right)$. We also get the analogue of (4.7), with the only difference that now we take into account all of the vertical one-dimensional slices and not only a subset of them.

Afterwards, we modify $\left(\tilde{v}_{k}\right)$ in those strips $L_{k}^{i}$ such that $\mathcal{H}^{1}\left(S_{v_{k}} \cap L_{k}^{i}\right)<3 / 4$ thus obtaining the final sequence $\left(w_{k}\right)$. The latter is defined replacing $\tilde{v}_{k}$ by a suitably chosen affine function, as in Theorem 2 Step 3. As before, the strong $L^{1}(Q)$-convergence to $u_{t}$ is preserved.

We notice that in this case there is at least one strip $L_{k}^{i}$ such that $\mathcal{H}^{1}\left(S_{v_{k}} \cap L_{k}^{i}\right) \geq 3 / 4$ (otherwise $w_{k}$ would be a Sobolev sequence with gradient uniformly bounded in $L^{2}\left(Q ; \mathbb{R}^{2}\right)$, thus violating the convergence to $u_{t}$ ). Moreover, if there are three or more of such strips, then $\mathcal{H}^{1}\left(S_{u_{k}} \cap Q\right) \geq 2$ and the lower bound (5.7) holds trivially true. We are then left with the case where there are one or two strips such that $\mathcal{H}^{1}\left(S_{v_{k}} \cap L_{k}^{i}\right)=\mathcal{H}^{1}\left(S_{w_{k}} \cap L_{k}^{i}\right) \geq 3 / 4$. Since $w_{k} \rightarrow u_{t}$ strongly in $L^{1}(Q)$, at least in one of the two strips as above we have

$$
\left|b_{k}^{j}-a_{k}^{j+h}\right| \geq \frac{t}{3}
$$

for $k$ large enough. We denote this strip by $L_{k}^{i *}$. As in Theorem 2 Step 4 , we now estimate from below $E_{\varepsilon_{k}}\left(u_{k}, Q\right)$ with the energy of $w_{k}$ in $L_{k}^{i^{*}}$. We find

$$
E_{\varepsilon_{k}}\left(u_{k}, Q\right) \geq \alpha \int_{L_{k}^{i^{*}}}\left|\nabla w_{k}\right|^{2} \mathrm{~d} x+\mathcal{H}^{1}\left(S_{w_{k}} \cap L_{k}^{i^{*}} \cap \varepsilon_{k} D_{\varepsilon_{k}}\right)+\frac{1}{\varepsilon_{k}} \mathcal{H}^{1}\left(\left(S_{w_{k}} \cap L_{k}^{i^{*}}\right) \backslash \varepsilon_{k} D_{\varepsilon_{k}}\right),
$$

where $\alpha$ is the constant given by Lemma 7 . 
Comparing the bulk energy of $w_{k}$ in $L_{k}^{i^{*}} \cap \varepsilon_{k} D_{\varepsilon_{k}}$ with that of the affine interpolation between the values $b_{k}^{j}$ and $a_{k}^{j+h}$, and using the same argument as in (4.12) we obtain

$$
\liminf _{k \rightarrow+\infty} \mathcal{H}^{1}\left(S_{w_{k}} \cap L_{k}^{i^{*}} \cap \varepsilon_{k} D_{\varepsilon_{k}}\right) \geq 1 \text {. }
$$

On the other hand, comparing the energy of $w_{k}$ in $L_{k}^{i^{*}} \backslash \varepsilon_{k} D_{\varepsilon_{k}}$ with those of the affine interpolation and of the piecewise constant function taking values in $\left\{b_{k}^{j}, a_{k}^{j+h}\right\}$ we get

$$
\alpha \int_{L_{k}^{i^{*}} \backslash \varepsilon_{k} D_{\varepsilon_{k}}}\left|\nabla w_{k}\right|^{2} \mathrm{~d} x+\frac{1}{\varepsilon_{k}} \mathcal{H}^{1}\left(\left(S_{w_{k}} \cap L_{k}^{i^{*}}\right) \backslash \varepsilon_{k} D_{\varepsilon_{k}}\right) \geq \min \left\{\frac{\alpha\left|b_{k}^{j}-a_{k}^{j+h}\right|^{2}}{(h-1)}, 1\right\} .
$$

Gathering the previous inequalities and the analogue of (4.10) gives

$$
\liminf _{k} E_{\varepsilon_{k}}\left(u_{k}\right) \geq \min \left\{1+\frac{\alpha \beta}{27} t^{2}, 2\right\},
$$

whence (5.7) follows. This concludes the proof of Theorem 5.

\section{REFERENCES}

[1] L. Ambrosio. Existence theory for a new class of variational problems, Arch. Rational Mech. Anal. 111 (1990), 291-322.

[2] L. Ambrosio, N. Fusco, and D. Pallara. Functions of bounded variation and free discontinuity problems. Oxford Mathematical Monographs. The Clarendon Press, Oxford University Press, New York, 2000.

[3] T.L. Anderson. Fracture Mechanics. Fundamentals and Applications, third edition. CRC Press, Taylor \& Francis, Boca Raton, 2005.

[4] M. Barchiesi and G. Dal Maso. Homogenization of fiber reinforced brittle materials: the extremal cases. SIAM J. Math. Anal. 41 (2009), 1874-1889.

[5] M. Barchiesi and M. Focardi. Homogenization of the Neumann problem in perforated domains: an alternative approach. Calc. Var. Partial Differential Equations 42 (2011), 257-288.

[6] G.I. Barenblatt. The mathematical theory of equilibrium cracks in brittle fracture. Adv. Appl. Mech. 7 (1962), $55-129$.

[7] G. Bouchitté, I. Fonseca, G. Leoni, and L. Mascarenhas. A global method for relaxation in $W^{1, p}$ and in $S B V_{p}$. Arch. Rational Mech. Anal. 165 (2002), 187-242.

[8] A. Braides. Approximation of free-discontinuity problems. Lecture Notes in Mathematics 1694. SpringerVerlag, Berlin, 1998.

[9] A. Braides. A handbook of $\Gamma$-convergence. In Handbook of Differential Equations: Stationary Partial Differential Equations, volume 3, pages 101-213. Elsevier, Amsterdam, 2006.

[10] A. Braides, G. Dal Maso, and A. Garroni. Variational formulation of softening phenomena in fracture mechanics: The one-dimensional case. Arch. Rational Mech. Anal. 146 (1999), 23-58.

[11] A. Braides, A. Defranceschi, and E. Vitali. Homogenization of free discontinuity problems. Arch. Rational Mech. Anal. 135 (1996), 297-356.

[12] A. Braides and L. Sigalotti. Models of defects in atomistic systems. Calc. Var. Partial Differential Equations 41 (2011), 71-109.

[13] A. Braides and M. Solci. Multi-scale free-discontinuity problems with soft inclusions. Boll. Unione Mat. Ital. (IX) 6 (2013), 29-51.

[14] F. Cagnetti and L. Scardia. An extension theorem in $S B V$ and an application to the homogenization of the Mumford-Shah functional in perforated domains. J. Math. Pures Appl. (9) 95 (2011), 349-381.

[15] S. Conti, M. Focardi, and F. Iurlano. Phase field approximation of cohesive fracture models. Ann. Inst. H. Poincaré Anal. Non Linéaire (2015), doi:10.1016/j.anihpc.2015.02.001.

[16] G. Cortesani. Strong approximation of $G S B V$ functions by piecewise smooth functions. Ann. Univ. Ferrara Sez. VII (N.S.), 43 (1997), 27-49.

[17] G. Dal Maso. An Introduction to $\Gamma$-convergence. Birkhäuser, Boston, 1993.

[18] G. Dal Maso, F. Iurlano. Fracture models as Gamma-limits of damage models. Commun. Pure Appl. Anal. 12 (2013), 1657-1686.

[19] G. Dal Maso, J.M. Morel, and S. Solimini. A variational method in image segmentation: Existence and approximation results. Acta Mathematica 168 (1992), 89-151. 
[20] G. Dal Maso, G. Orlando, and R. Toader. Fracture models for elasto-plastic materials as limits of gradient damage models coupled with plasticity: the antiplane case. To appear in Calc. Var. Partial Differential Equations.

[21] G. Dal Maso and C.I. Zeppieri. Homogenization of fiber reinforced brittle materials: the intermediate case. Adv. Calc. Var. 3 (2010), 345-370.

[22] M. Focardi, M.S. Gelli, and M. Ponsiglione. Fracture mechanics in perforated domains: a variational model for brittle porous media. Math. Models Methods Appl. Sci. 19 (2009), 2065-2100.

[23] A. Giacomini. Size effects on quasi-static growth of cracks. SIAM J. Math. Anal. 36 (2005), 1887-1928.

(Marco Barchiesi) Dipartimento di Matematica ed Applicazioni, Università di Napoli "Federico II", Via Cintia, 80126 Napoli, Italy

E-mail address: barchies@gmail.com

(Giuliano Lazzaroni) SISSA, Via Bonomea 265, 34136 Trieste, Italy

E-mail address: giuliano.lazzaroni@sissa.it

(Caterina Ida Zeppieri) Institut für Numerische und Angewandte Mathematik, Universität Münster, Einsteinstr. 62, 48149 Münster, GERMAnY

E-mail address: caterina.zeppieri@uni-muenster.de 